STRUCTURAL BIOLOGY

ISSN 2059-7983

\section{Structural insights into the interaction of the conserved mammalian proteins GAPR-1 and Beclin 1, a key autophagy protein}

\author{
Yue Li, ${ }^{\text {a }}$ Yuting Zhao, ${ }^{\text {b }}$ Minfei Su, ${ }^{\text {a }}$ Karen Glover, ${ }^{\text {a }}$ Srinivas Chakravarthy, \\ Christopher L. Colbert, ${ }^{a}$ Beth Levine ${ }^{b}$ and Sangita C. Sinha ${ }^{a} *$
}

Received 18 April 2017

Accepted 14 August 2017

Edited by R. McKenna, University of Florida, USA

Keywords: GAPR-1; CAP domain; autophagy; Beclin 1; BECN1; protein-protein interactions.

PDB reference: pentad mutant of GAPR-1, 5vhg

Supporting information: this article has supporting information at journals.iucr.org/d

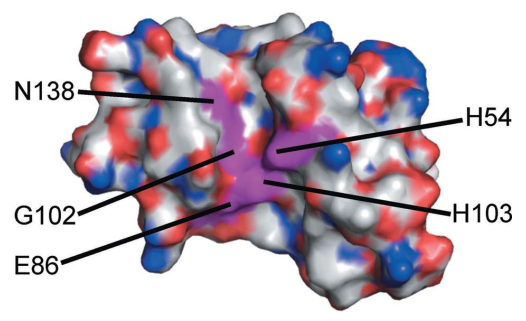

(C) 2017 International Union of Crystallography a Department of Chemistry and Biochemistry, North Dakota State University, Fargo, ND 58108, USA, ${ }^{\mathbf{b}}$ Center for Autophagy Research, Department of Internal Medicine and Microbiology, UT Southwestern Medical Center and Howard Hughes Medical Institute, Dallas, TX 75390, USA, and 'Bio-CAT, Advanced Photon Source, 9700 South Cass Avenue, LSEP Building 435B, Argonne, IL 60439, USA. *Correspondence e-mail: sangita.sinha@ndsu.edu

Mammalian Golgi-associated plant pathogenesis-related protein 1 (GAPR-1) is a negative autophagy regulator that binds Beclin 1, a key component of the autophagosome nucleation complex. Beclin 1 residues 267-284 are required for binding GAPR-1. Here, sequence analyses, structural modeling, mutagenesis combined with pull-down assays, $\mathrm{X}$-ray crystal structure determination and small-angle X-ray scattering were used to investigate the Beclin 1-GAPR-1 interaction. Five conserved residues line an equatorial GAPR-1 surface groove that is large enough to bind a peptide. A model of a peptide comprising Beclin 1 residues 267-284 docked onto GAPR-1, built using the CABS-dock server, indicates that this peptide binds to this GAPR-1 groove. Mutation of the five conserved residues lining this groove, H54A/E86A/G102K/H103A/N138G, abrogates Beclin 1 binding. The $1.27 \AA$ resolution X-ray crystal structure of this pentad mutant GAPR-1 was determined. Comparison with the wild-type (WT) GAPR-1 structure shows that the equatorial groove of the pentad mutant is shallower and more positively charged, and therefore may not efficiently bind Beclin 1 residues 267-284, which include many hydrophobic residues. Both WT and pentad mutant GAPR-1 crystallize as dimers, and in each case the equatorial groove of one subunit is partially occluded by the other subunit, indicating that dimeric GAPR-1 is unlikely to bind Beclin 1. SAXS analysis of WT and pentad mutant GAPR-1 indicates that in solution the WT forms monomers, while the pentad mutant is primarily dimeric. Thus, changes in the structure of the equatorial groove combined with the improved dimerization of pentad mutant GAPR-1 are likely to abrogate binding to Beclin 1.

\section{Introduction}

Golgi-associated plant pathogenesis-related protein (GAPR-1), also known as GLIPR-2 or C9orf19, is a mammalian protein that belongs to the cysteine-rich secretory proteins, antigen 5 and pathogenesis-related (CAP) protein superfamily (Eberle et al., 2002). CAP superfamily proteins are found in diverse organisms ranging from bacteria to humans (Gibbs et al., 2008). Mammals have multiple CAP domain-containing proteins. Most CAP-domain proteins are secreted outside the cell, where they have endocrine and paracrine functions (Gibbs et al., 2008). CAP proteins are implicated in diverse biological functions such as reproduction, immune regulation and tumor suppression.

The CAP domain is characterized by a molecular mass of $17-21 \mathrm{kDa}$ and a conserved $\alpha-\beta-\alpha$ sandwich fold, wherein the central $\beta$-sheet is flanked by three helices on one side and by the fourth helix on the other side, with the sandwich fold stabilized by hydrophobic packing, hydrogen bonds and 
disulfide bonds (Fernández et al., 1997; Henriksen et al., 2001; Serrano et al., 2004; Asojo et al., 2005, 2011; Suzuki et al., 2008; Asojo, 2011; Osman et al., 2012; Mason et al., 2014; Darwiche et al., 2016; Olrichs \& Helms, 2016). Most members of this superfamily have a signal peptide at the $\mathrm{N}$-terminus and various additional $\mathrm{C}$-terminal domains that are likely to assign the CAP domain to a specific biological function (Gibbs et al., 2008). Based on sequence similarity, four conserved sequence motifs named CAP1-CAP4 have been identified in CAP domains (Gibbs et al., 2008).

Multiple, diverse molecular mechanisms have been attributed to different CAP domains. CAP domains have been shown to bind metals (Henriksen et al., 2001; Suzuki et al., 2008; Wang et al., 2010; Asojo et al., 2011; Osman et al., 2012; Mason et al., 2014; Darwiche et al., 2016), cholesterol (Choudhary \& Schneiter, 2012; Choudhary et al., 2014; Kelleher et al., 2014; Darwiche et al., 2016), other lipids or lipid-like molecules (van Galen et al., 2012; Xu et al., 2012; Darwiche et al., 2016) and peptides (Milne et al., 2003; Mason et al., 2014), and are also predicted to bind glycans (Osman et al., 2012). Most CAP domains have an equatorial surface groove that, at its center, often includes a deeper cavity lined by conserved residues. This equatorial surface groove has been shown to bind metal ions, often within the deeper central cavity (Henriksen et al., 2001; Suzuki et al., 2008; Wang et al., 2010; Asojo et al., 2011; Mason et al., 2014; Darwiche et al., 2016). The equatorial surface groove is also predicted to bind peptides (Milne et al., 2003; Osman et al., 2012; Mason et al., 2014) or other large molecules such as glycans (Osman et al., 2012). Notably, the CAP domain of the cone snail venom Tex31 has protease activity and can efficiently bind and cleave the conotoxin propeptide (Milne et al., 2003). In contrast, the yeast Pry1 CAP domain is required and sufficient for binding and transporting cholesterol (Choudhary \& Schneiter, 2012; Choudhary et al., 2014). A pocket on the Pry1 CAP domain, distinct from the equatorial groove and named the caveolinbinding motif, binds 1,4-dioxane from the crystallization solution (Darwiche et al., 2016). The caveolin-binding motif is also required for cholesterol binding, as mutagenesis of these residues abrogates in vivo sterol export (Choudhary et al., 2014). However, none of these molecular mechanisms are observed in a wide range of CAP domains, nor have they been characterized in detail. Thus, the molecular mechanism(s) of CAP domains is/are still not well understood.

GAPR-1 is highly expressed in heart, lung and peripheral blood leukocytes, and at lower levels in skeletal muscle, prostate and uterus (Baxter et al., 2007). GAPR-1 is unique amongst mammalian CAP domain-containing proteins in that it consists of only the CAP domain and lacks a signal peptide (Eberle et al., 2002; Serrano et al., 2004). Consistent with the absence of a signal peptide, and unlike other CAP-domain proteins, GAPR-1 lacks disulfide bonds and is not secreted by canonical pathways, although some reports indicate that it might be secreted into the lumen of small vesicles in seminal fluid by a noncanonical secretory pathway (Van Loon \& Van Strien, 1999; Eberle et al., 2002; Aalberts et al., 2012). Further, GAPR-1 localizes to lipid-rich microdomains of the cytosolic leaflet of the Golgi complex. It binds tightly to negatively charged lipid head-groups such as inositol phosphate ( $\mathrm{IP}_{6}$ ) (van Galen et al., 2012). The crystal structure of GAPR-1 shows that, like other CAP domains, it has a wide and long equatorial groove, but the function of this groove has not yet been established. GAPR-1 was crystallized as a dimer, but appears to equilibrate between monomeric and dimeric states in solution (Serrano et al., 2004). Yeast two-hybrid assays suggest that GAPR-1 may exist as a dimer in cells (Serrano et al., 2004). However, another study suggests that GAPR-1 is a monomer in the absence of lipids (van Galen et al., 2012). The presence of negatively charged lipids appears to alter the GAPR-1 dimer, with one GAPR-1 subunit rotating by $28.5^{\circ}$ relative to the other (van Galen et al., 2012). Moreover, incubation with liposomes composed of negatively charged lipids stabilizes GAPR-1 dimers, and prolonged incubation causes the formation of amyloid-like fibrils via $\beta$-sheet extension (Olrichs et al., 2014). Thus, the oligomeric state of GAPR-1 has not been unambiguously established and may change in different cellular contexts.

GAPR-1 has been shown to down-regulate autophagy and bind to a key autophagy protein, Beclin 1 (Shoji-Kawata et al., 2013). Autophagy is a lysosomal degradation pathway that is essential for cellular homeostasis, differentiation and development (Mizushima, 2005; Rubinsztein et al., 2011; Mizushima \& Komatsu, 2011). Therefore, dysfunction of autophagy is related to many diseases, such as cancers, neurodegenerative diseases, cardiovascular diseases and infectious diseases (Levine \& Kroemer, 2008; Mizushima et al., 2008; Levine et al., 2011; Rubinsztein et al., 2012; Choi et al., 2013). Beclin 1 is an essential component of the vacuolar protein sorting 34 (Vps34) complexes that up-regulate autophagy (Levine \& Klionsky, 2004; Levine et al., 2015). Many proteins, such as various Bcl-2 homologs, are implicated in regulating autophagy by binding to Beclin 1 and modulating its function (Pattingre et al., 2005; Maiuri et al., 2007; Sinha \& Levine, 2009; Hatok \& Racay, 2016). Beclin 1 consists of an intrinsically disordered region, a flexible helical domain, a coiled-coil domain and a $\beta-\alpha$ repeat in autophagy domain (BARAD) (Mei et al., 2016). Beclin 1 co-immunoprecipitates GAPR-1, and deletion of Beclin 1 residues 267-284, which map to the BARAD, diminishes GAPR-1 co-immunoprecipitation (Shoji-Kawata et al., 2013). Further, a cell-permeable peptide derived from Beclin 1 residues 267-284 is sufficient to pull down GAPR-1 and has been shown to induce autophagy, possibly by competitively preventing GAPR-1 from binding to Beclin 1. Peptides with Beclin 1 F270S or F274S mutations fail to bind to GAPR-1 and fail to induce autophagy (ShojiKawata et al., 2013).

The mechanism by which GAPR-1 binds to Beclin 1 to down-regulate autophagy is unknown. We hypothesized that the GAPR-1 equatorial surface groove, which is lined with five conserved residues, may be responsible for binding Beclin 1 . In order to better understand the interaction of GAPR-1 and Beclin 1, we constructed a model of the GAPR-1-Beclin 1 (residues 267-284) complex to identify potential GAPR-1 residues involved in the GAPR-1-Beclin 1 interaction and 
used pull-down assays to test the impact of mutating these residues on the binding of GAPR-1 to Beclin 1. We solved the high-resolution three-dimensional structure of a mutant GAPR-1 that does not bind to Beclin 1 to elucidate structural changes that are likely to disrupt binding. Lastly, we used isothermal titration calorimetry (ITC) to quantify the selfdissociation constants of WT and mutant GAPR-1 and used size-exclusion chromatography together with small-angle $\mathrm{X}$-ray scattering (SEC-SAXS) to verify the oligomeric states of wild-type (WT) and mutant GAPR-1 in solution.

\section{Materials and methods}

2.1. Constructing a model of the complex of GAPR-1 and Beclin 1 residues 267-284

Sequence alignments were performed using ClustalW2 (Thompson et al., 1994). The docking server CABS-dock (Kurcinski et al., 2015) was used to model the binding of Beclin 1 residues 267-284 to the 'receptor molecule', i.e. WT GAPR-1 (PDB entry 1smb; Serrano et al., 2004). Docking involved three discrete steps. (i) Random structures of a peptide comprising Beclin 1 residues 267-284 were generated and placed on the surface of the receptor. (ii) Replica exchange Monte Carlo dynamics was used in docking, yielding a total of 10000 models of the GAPR-1-Beclin 1 residues 267-284 complex. (iii) The final representative models were selected in two substeps: (1) all unbound states were excluded and the 100 lowest binding energy models of the GAPR-1-Beclin 1 residues 267-284 complex were selected for the next step; (2) selected models were clustered based on the root-meansquare deviation (r.m.s.d.) of the entire complex for model refinement; the clusters were ranked according to size from largest to the tenth largest and the ten top-ranked models (representatives of the ten most populated clusters) were selected.

\subsection{Creation of cellular protein-expression constructs,} pull-down assay and Western blot

WT GAPR-1 residues 1-154 were subcloned from GAPR-1Myc (Shoji-Kawata et al., 2013) using the primers 5'-GAA GATCTATGGGCAAGTCAGCTTCCA and 5'-TTGCGG CCGCTTACTTCTTCGGCGGCAGGA into a pGEX-6P-1 vector between the BamHI and NotI restriction-enzyme sites. GAPR-1 mutants E86A, G102K/H103A, H54A/G102K/ H103A/N138G, E86A/G102k/H102A/N138G and H54A/ E86A/G102K/H103A/N138G were generated by PCR mutagenesis using the QuikChange II Site-Directed Mutagenesis Kit (Agilent Technologies).

WT Beclin 1 residues 1-450 were subcloned from FLAGBeclin 1 (Shoji-Kawata et al., 2013) using primers 5'-CGG GATCCATGGAAGGGTCT and 5'-TTGCGGCCGCTCATT TGTTATAAAATTGTG between the BamHI and NotI restriction-enzyme sites of a pGEX-4T-1 vector.

For GST pull-down assays, the GST fusion proteins described above were expressed in Escherichia coli strain BL21-CodonPlus(DE3)-RP and purified using glutathione
Sepharose 4B (GE Healthcare). GST-tagged GAPR-1 was treated with PreScission protease to remove the GST tag. GAPR-1 variants were incubated with GST-Beclin 1 or GST conjugated to glutathione Sepharose 4B beads in binding buffer (PBS with $0.5 \%$ Triton X-100). After a $2 \mathrm{~h}$ incubation at $4{ }^{\circ} \mathrm{C}$, the beads were washed three times with binding buffer. The input and bound GAPR-1 proteins were analysed by Western blotting using an anti-GAPR-1 antibody (Eberle et al., 2002). The amount of GST or GST-Beclin 1 pulled down was analysed by staining the same polyvinylidene fluoride membrane with Ponceau S stain.

\subsection{Large-scale protein expression and purification}

For large-scale protein purification, the plasmids described above, encoding either WT or H54A/E86A/G102K/H103A/ N138G pentad mutant GAPR-1 (hereafter called the pentad mutant), were used to transform E. coli BL21(DE3) pLysS cells, which were grown at $37^{\circ} \mathrm{C}$ to $\mathrm{OD}_{600}=0.8$ (log phase) prior to the induction of protein expression with $0.5 \mathrm{~m} M$ IPTG at $20^{\circ} \mathrm{C}$ for $18 \mathrm{~h}$. The cells were harvested via centrifugation at $4000 \mathrm{~g}$ for $20 \mathrm{~min}$ and the cell pellets were frozen at $-80^{\circ} \mathrm{C}$. The frozen pellets were thawed and resuspended in $300 \mathrm{ml}$ lysis buffer ( $25 \mathrm{~m} M$ HEPES pH 7.5, $300 \mathrm{mM} \mathrm{NaCl}, 10 \%$ glycerol, $2 \mathrm{~m} M$ DTT) with $0.2 \mathrm{~m} M$ AEBSF, and the resuspended cells were lysed in a NanoDeBEE emulsifier (BEE International). The lysate was centrifuged at $20000 \mathrm{~g}$ for $40 \mathrm{~min}$ to pellet the cell debris. The protein was purified from the clarified lysate by GST affinity chromatography (GE Healthcare), followed by overnight on-column cleavage using GST PreScission protease to remove the GST tag. This cleavage leaves a linker comprising residues GPLGS on the protein. The cleaved protein was washed with two column volumes of lysis buffer, purified further by cation-exchange chromatography using an $8 \mathrm{ml}$ HR10/10 Mono S column (GE Healthcare) and finally by size-exclusion chromatography (SEC) using a HiLoad 16/60 Superdex 200 column (GE Healthcare). The final purified protein was concentrated to $1 \mathrm{~m} M$ in a buffer comprised of $50 \mathrm{~m} M$ Tris $\mathrm{pH} 8.0,300 \mathrm{~m} M$ $\mathrm{NaCl}, 2 \mathrm{~m} M$ DTT and stored at $-80^{\circ} \mathrm{C}$.

\subsection{Crystallization, data collection and structure solution}

The pentad GAPR-1 mutant was crystallized at $20^{\circ} \mathrm{C}$ by hanging-drop vapor diffusion, wherein the drop consisted of $1.5 \mu \mathrm{l}$ protein stock $\left(17 \mathrm{mg} \mathrm{ml}^{-1}\right.$ protein in $50 \mathrm{mM}$ Tris $\mathrm{pH} 8.0$, $300 \mathrm{mM} \mathrm{NaCl}, 2 \mathrm{~m} M$ DTT) and $0.5 \mu \mathrm{l}$ reservoir solution

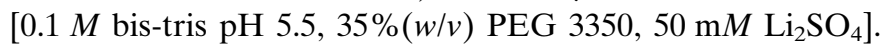
Crystals grew within a week and were harvested in LV CryoOil (MiTeGen) before being flash-cooled in liquid nitrogen.

Diffraction data were collected at $100 \mathrm{~K}$ at a wavelength of $0.97918 \AA$ in one $360^{\circ}$ sweep from a single crystal on NE-CAT beamline 24-ID-C at the Advanced Photon Source (APS), Argonne National Laboratory (ANL), Argonne, Illinois, USA. Diffraction intensities were recorded on a $4 \times 4$ tiled MAR Mosaic CCD detector (Rayonix) at a crystal-to-detector distance of $250 \mathrm{~mm}$ over a $0.5^{\circ}$ crystal rotation with $1 \mathrm{~s}$ exposure per image. Diffraction data were processed using 
Table 1

X-ray data-collection and structure-refinement statistics.

Values in parentheses are for the highest resolution shell.

\begin{tabular}{ll}
\hline Data-collection statistics & $C 222_{1}$ \\
Space group & $a=62.3, b=84.7, c=51.4$ \\
Unit-cell parameters $(\AA)$ & $51.43-1.27$ \\
Resolution $(\AA)$ & 9.94 \\
Wilson $B$ factor $\left(\AA^{2}\right)$ & $35987(3588)$ \\
Unique reflections & $93.8(99.5)$ \\
Completeness $(\%)$ & $3.4(3.3)$ \\
Multiplicity & $11(2.1)$ \\
$\langle I / \sigma(I)\rangle$ & $0.986(0.742)$ \\
C $C_{1 / 2}$ & \\
Refinement statistics & $5 \mathrm{vhg}$ \\
PDB code & $25.71-1.27(1.32-1.27)$ \\
Resolution range & $99(98)$ \\
Completeness $(\%)$ & $14.5(20.8)$ \\
$R_{\text {work }} \dagger(\%)$ & $17.4(24.6)$ \\
$R_{\text {free }}^{\dagger}$ (\%) & 151 \\
No. of protein residues & 222 \\
No. of waters & 0.009 \\
R.m.s.d., bond lengths $(\AA)$ & 0.97 \\
R.m.s.d., angles $\left({ }^{\circ}\right)$ & 0 \\
Ramachandran outliers $(\%)$ & \\
Average $B$ factor $\left(\AA^{2}\right)$ & 16.03 \\
Overall & 13.75 \\
Macromolecules & 29.18 \\
Solvent &
\end{tabular}

$\dagger R$ factor $=\sum_{h k l}|| F_{\text {obs }}|-| F_{\text {calc }}|| / \sum_{h k l}\left|F_{\text {obs }}\right| . \quad \ddagger$ The test set for $R_{\text {free }}$ consisted of $5 \%$ of the data.

the RAPD automated processing web server (https:// rapd.nec.aps.anl.gov/rapd/). Data statistics are shown in Table 1 . The structure was solved by molecular replacement using Phaser (McCoy et al., 2007) in the PHENIX suite (Adams et al., 2010), with the WT GAPR-1 structure (PDB entry $1 \mathrm{smb}$ ) as a model. The structure was refined to $1.27 \AA$ resolution using the PHENIX refinement pipeline (Adams et al., 2010) combined with iterations of manual model building using Coot (Emsley et al., 2010). Final model statistics are reported in Table 1 .

\subsection{Protein structure analysis}

Pairwise superimposition of structures was performed with LSQKAB (Kabsch et al., 1976). Simultaneous superimposition of multiple structures was performed with THESEUS (Theobald \& Wuttke, 2008), which applies a maximumlikelihood method. Electrostatic properties were calculated with APBS (Baker et al., 2001) using the PARSE force field, and the electrostatic potentials were compared using the webPIPSA server (Richter et al., 2008). Dimer interfaces in each crystal structure were analysed in detail using PISA (Krissinel \& Henrick, 2007). Shape complementarity at the WT and pentad mutant dimer interfaces was calculated using the program $S C$ in the CCP4 suite (Lawrence \& Colman, 1993; Winn et al., 2011). Molecular figures were created using PyMOL (v.1.5.0.2; Schrödinger).

\subsection{Analytical SEC}

Analytical SEC was performed to estimate and compare the molecular mass of WT and pentad mutant GAPR-1. $0.2 \mathrm{ml}$ of $2,1,0.5$ and $0.25 \mathrm{~m} M$ purified WT or pentad mutant GAPR-1 in $50 \mathrm{~m} M$ Tris $\mathrm{pH} 8.0,300 \mathrm{~m} M \mathrm{NaCl}, 2 \mathrm{~m} M$ DTT was injected onto a Superdex 200 10/300 SEC column (GE Healthcare) and the molecular masses and hydrodynamic (Stokes) radii $\left(R_{\mathrm{S}}\right)$ were calculated by comparison with the elution profile of SEC standards (Bio-Rad).

\subsection{ITC}

ITC experiments were performed using a Low Volume Nano ITC (TA Instruments). WT and pentad mutant GAPR-1 samples were dialyzed against ITC buffer comprising $50 \mathrm{~m} M$ Tris $\mathrm{pH} 8.0,300 \mathrm{~m} M \mathrm{NaCl}, 2 \mathrm{~m} M \beta$-mercaptoethanol. All ITC experiments were performed at $20^{\circ} \mathrm{C}$ with 25 injections of $2 \mu \mathrm{l}$ each, by titrating $0.26 \mathrm{mM}$ protein into ITC buffer. Blank profiles obtained by titrating buffer into buffer were subtracted from the experimental profiles of self-dissociation experiments. Data were analyzed with the NanoAnalyze software (TA Instruments) and fitted with dimer dissociation models. The data reported are the average of three sets of experiments.

\subsection{SEC-SAXS data collection and analysis}

SAXS data were collected on beamline 18-ID (BioCAT) at APS, ANL, Argonne, Illinois, USA using a PILATUS3 1M detector with X-rays of wavelength $1.033 \AA$ A. $17.2 \mathrm{mg} \mathrm{ml}^{-1}$ WT GAPR-1 or pentad mutant GAPR-1 was injected onto a Superdex 200 10/300 SEC (GE Healthcare) column at a flow rate of $0.75 \mathrm{ml} \mathrm{min} \mathrm{m}^{-1}$ and the eluate from the SEC column was immediately exposed to the X-ray beam for $1 \mathrm{~s}$, with a $2 \mathrm{~s}$ delay between each exposure. The SAXS data were recorded at a sample-to-detector distance of $3.5 \mathrm{~m}$, which covers a momentum-transfer range from 0.0036 to $0.4 \AA^{-1}[q=$ $(4 \pi \sin \theta) / \lambda$, where $2 \theta$ is the scattering angle]. Scattering data were normalized to the incident X-ray beam intensity prior to further analysis. Singular value decomposition (SVD) analysis was performed on the normalized scattering data using the program DELA (Lambright et al., 2014; Malaby et al., 2015). The scattering from buffer was subtracted using the BioCAT beamline pipeline based on the ATSAS suite of programs. A $q$ range of $q<0.3 \AA$ was used for all SAXS analyses using the $A T S A S$ program suite (Franke et al., 2017). Within the ATSAS program suite, PRIMUS (Konarev et al., 2003) was used to scale and average data for further analysis, and also for Guinier extrapolation. The radius of gyration $\left(R_{\mathrm{g}}\right)$ of the averaged data was calculated from the Guinier approximation. The particle pairwise distance distribution function $[P(r)$ plot $]$ and Kratky plot were calculated using GNOM (Svergun, 1992). The $P(r)$ plot was used to estimate $R_{\mathrm{g}}$ and $D_{\max }$, which were used to constrain the dimensions of the ten independent $a b$ initio bead models calculated using DAMMIF (Franke \& Svergun, 2009). $P 1$ symmetry was imposed to generate these models. A total of ten $a b$ initio reconstructed models were further averaged and filtered by DAMSEL, DAMSUP, $D A M A V E R$ and DAMFILT (Volkov \& Svergun, 2003). CRYSOL (Svergun et al., 1995) was used to calculate and compare theoretical scattering curves from either the WT (PDB entry 1smb) or pentad mutant GAPR-1 structures. 
GAPR1

GAPR1

GLIPR-1

Pseudechetoxin

CRVP

VAL4

P14a

PRY1

$\alpha 1$

bellelelelelele

MGKSASKQF HNEVIKA HNEYRQKH . . . . G . VPP I

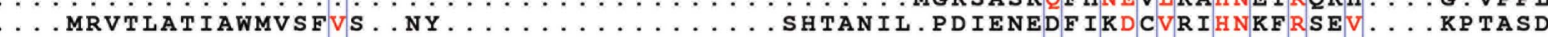

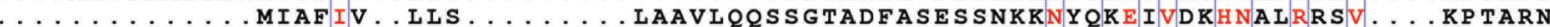
... ME ISGLVYLI I IVT I I DLPYGKA /18aa/CKYGS LKPNCGNKVVV SYGITKOEKODITKEHNDFROKI/12aa/ OPRAKN TYV * .

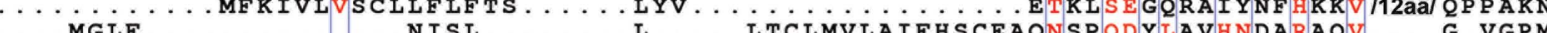

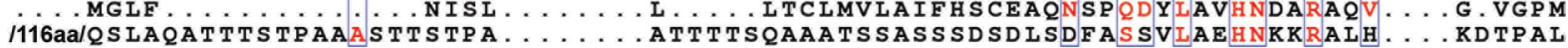

GAPR1
GAPR1
GLIPR-1
Pseudechetoxin
CRVP
VAL4
P14a
PRY1

$$
\underset{30}{\beta 1}{ }_{40}^{\alpha 2}
$$

$\alpha 3$

. . . . . SSRGOCGENIAWASYD . . . OTGKEVADRWYSEIKNYNFOOPGETS. SNCOF SHNTRLKP P K KHPNETSLGENIWTGSVP... IFSVSSA ITNWYDEIODYDFK..TRICK MLYMTWDPALAOIAKAWA

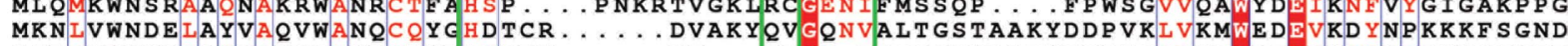
LTKLKWNKL LANKAKQQAKRCKYDSNDPN ....... DF... I I GD. FES I GQNLADYPTIEGAMKDWLEEYKNYNFEKNQCNGD

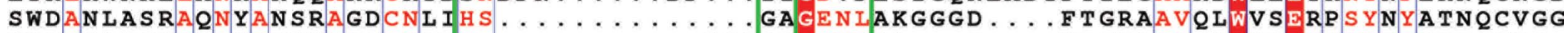

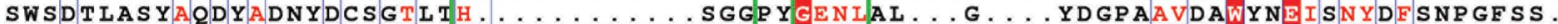

\section{CAP3}

GAPR1
GAPR1
GLIPR-1
P seudechetoxin
CRVP
VAL4
P14a
PRY1

Figure 1

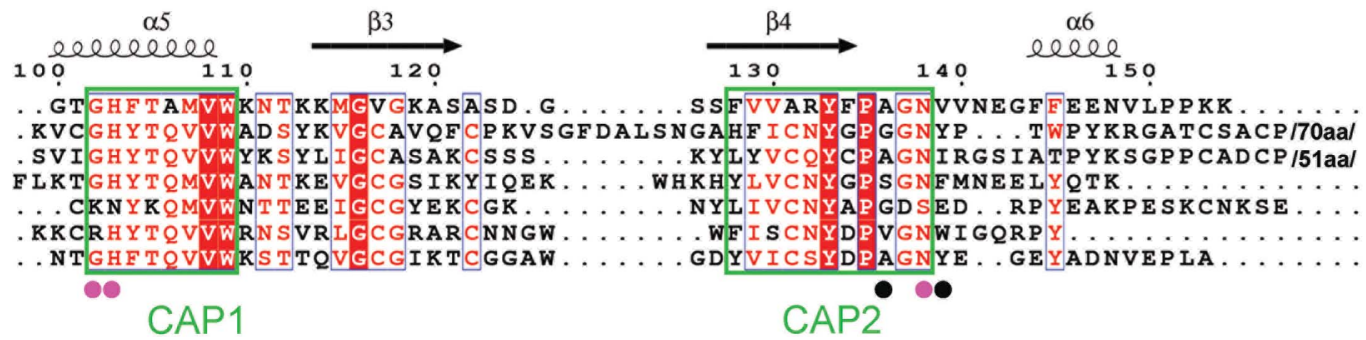

GAPR-1 sequence conservation. Alignment of GAPR-1 homolog sequences from different species: GAPR-1, Homo sapiens; GLIPR-1, Homo sapiens; pseudechetoxin, Pseudechis australis; CRVP, Vespula vulgaris; VAL4, Schistosoma mansoni; P14a, Solanum lycopersicum; PRY1, Saccharomyces cerevisiae. Conserved residues are highlighted in red and invariant residues in white on a red background. Secondary-structure elements, i.e. helices and strands, displayed as spirals and arrows, respectively, above the alignment correspond to WT GAPR-1. The four conserved CAP sequence motifs are boxed in green. Circles below the sequence alignment indicate residues lining the equatorial binding groove, with magenta circles indicating conserved residues mutated in this study. This figure was made with ESPript (Robert \& Gouet, 2014).

Superimposition of the crystal structures on the $a b$ initio bead models was performed using SUPCOMB (Kozin \& Svergun, 2001).

\section{Results}

3.1. Beclin 1 residues $267-284$ are predicted to bind to the conserved equatorial groove of the GAPR-1 CAP domain

Several conserved GAPR-1 residues were identified from an alignment of six CAP protein sequences from different species: GLIPR-1 from human, pseudechetoxin from king brown snake, CRVP from common wasp, VAL4 from blood fluke, P14a from tomato and Pry1 from yeast (Fig. 1). The equatorial surface groove on the GAPR-1 surface is the only groove that is large enough to accommodate a peptide. This groove is lined by 11 residues, six of which (His54, Glu65, Glu86, Gly102, His103 and Asn138) are conserved amongst various GAPR-1 orthologs (Figs. 1 and 2). The Glu65 mainchain atoms are surface-exposed, but the side chain is buried in the core of the protein, with the side-chain carboxylate stabilized by a bidentate salt bridge to the Arg132 guanidinium and by hydrogen bonds to the Ser55 hydroxyl and mainchain amide. Thus, Glu65 and equivalent residues of homologs are likely to be essential for the structural integrity of CAP domains rather than peptide binding. The side chains of the remaining five residues are solvent-exposed, and amongst these His54 is part of the CAP3 motif, Gly102 and His103 are part of the CAP1 motif and Asn138 is part of the CAP2 motif. Notably, Beclin 1, including residues 267-284, is also conserved across species from yeast to humans. Thus, we hypothesized that the five conserved, solvent-exposed GAPR-1 residues lining the equatorial groove may play essential roles in binding to a conserved region of Beclin 1.

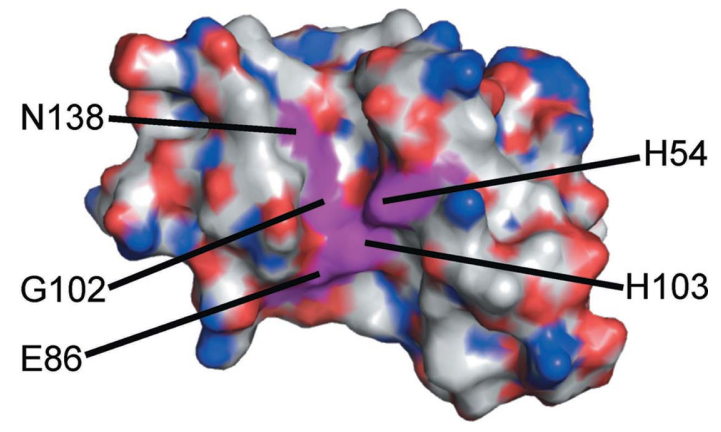

Figure 2

Location of mutated, conserved residues within the equatorial GAPR-1 binding groove. The molecular surface of GAPR-1 is colored by atom type: oxygen, red; nitrogen, blue; carbon, gray; mutated residues are colored magenta. This and all other molecular figures were made with PyMOL. 
The program $C A B S$-dock (Kurcinski et al., 2015) was used to dock a peptide corresponding to Beclin 1 residues 267-284 onto the 'receptor molecule', i.e. WT GAPR-1 (PDB entry 1smb; Fig. 3). $C A B S$-dock does not require a priori information about the binding site and allows complete peptide flexibility and small fluctuations of the 'receptor' backbone during the docking search. The top-ranked model cluster of the WT GAPR-1-Beclin 1 (residues 267284) complex (Fig. 3) has an r.m.s.d. of $6.39 \AA$ over 18 peptide $\mathrm{C}^{\alpha}$ atoms. The model from the top-ranked model cluster suggests that Beclin 1 residues 267-284 bind to the equatorial groove on the GAPR-1 CAP domain (Fig. 3). The docked peptide superimposes on equivalent residues of the Beclin 1 BARAD structure (PDB entry 4ddp; Huang et al., 2012) with an r.m.s.d. of $4.4 \AA$ over $16 \mathrm{C}^{\alpha}$ atoms.

3.2. Conserved GAPR-1 residues lining the equatorial binding groove are important for binding to Beclin 1

The role of the five conserved GAPR-1 residues whose side chains line the equatorial groove (Fig. 2) was evaluated by assessing the impact of five sets of GAPR-1 mutations (E86A, G102K/H103A double, H54A/G102K/ H103A/N138G tetrad, E86A/G102K/ H103A/N138G tetrad and H54A/E86A/ G102K/H103A/N138G pentad mutants) on the GAPR-1-Beclin 1 interaction, as monitored by GST pull-down assays (Fig. 4). These assays show that relative to WT GAPR-1, each of these GAPR-1 mutants has substantially decreased binding to Beclin 1, indicating that the residues within each set of mutations are likely to contribute to the Beclin 1 interaction interface. Binding to Beclin 1 is not abolished by either the E86A single mutant or the G102K/H103A double mutant (Fig. 4), or the N138G single mutant (data not shown), indicating that individually none of these residues is critical for binding. However, the E86A/G102K/H103A/N138G tetrad and H54A/E86A/G102K/H103A/ N138G pentad mutations completely abrogate the GAPR-1-Beclin 1 interaction, suggesting that together the conserved residues lining the equatorial
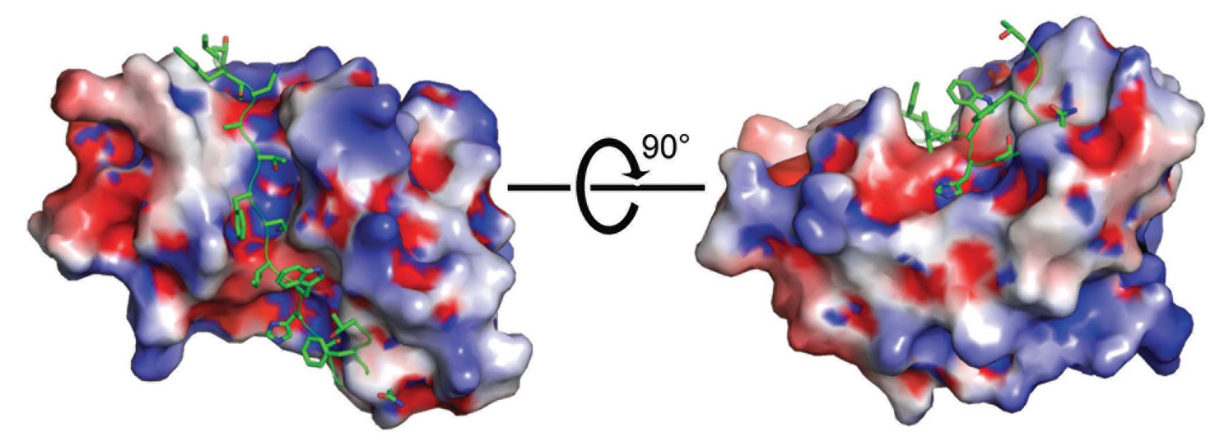

Figure 3

Computational model of Beclin 1 (residues 267-284) docked onto WT GAPR-1 (PDB entry 1smb). The electrostatic potential surface of GAPR-1 is shown and the docked peptide comprising Beclin 1 (residues 267-284) is displayed in green, with side chains shown in stick representation colored by atom type: oxygen, red; nitrogen, blue; carbon, green. The range of electrostatic potential displayed is -5 to $5 k T$.
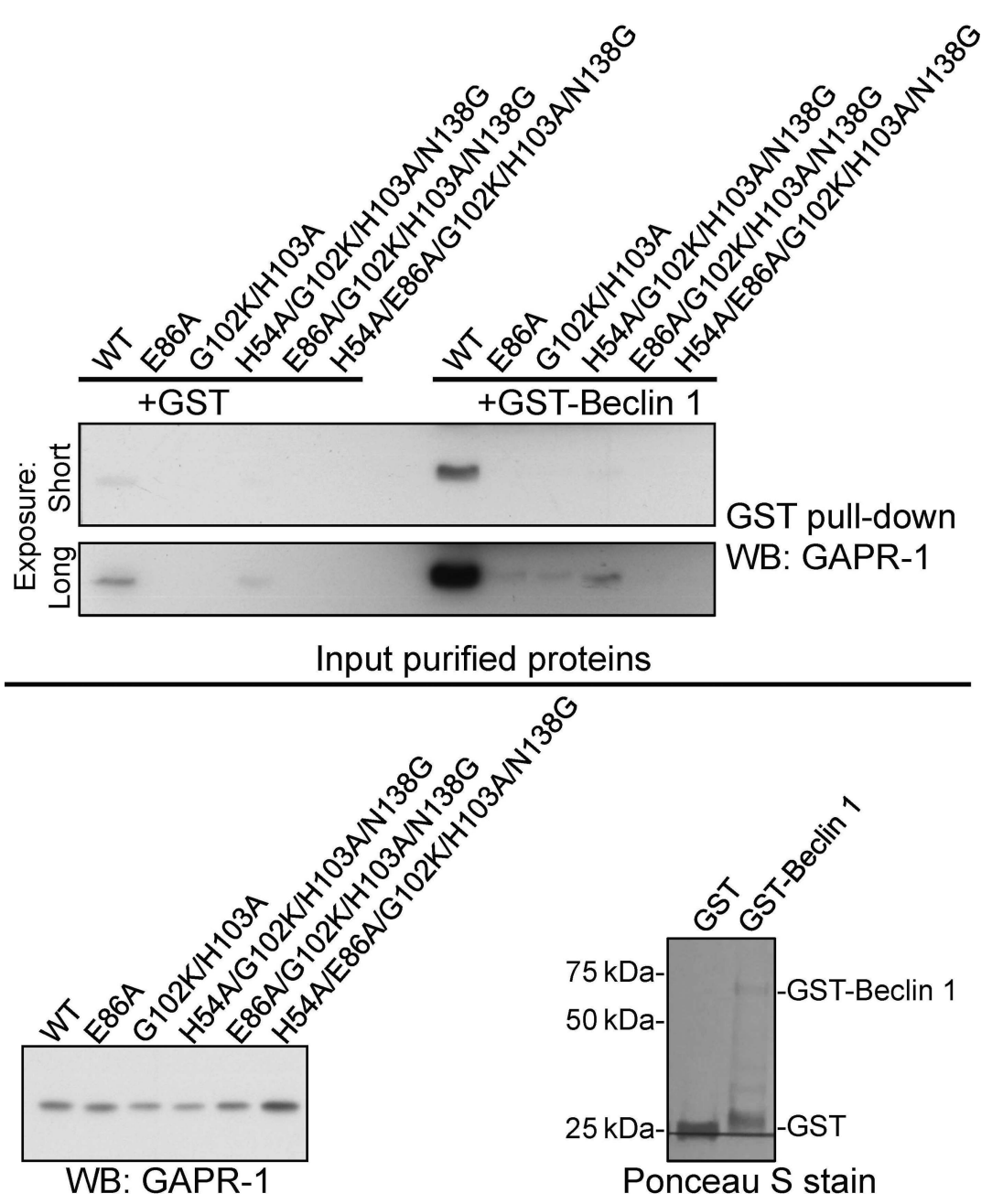

Figure 4

GST-Beclin 1 pull-downs of WT and mutant GAPR-1. GST-tagged Beclin 1 was used as the bait to pull down WT and mutant GAPR-1. The GAPR-1 bound to Beclin 1 was detected by anti-GAPR-1 antibodies. WB: Western blot. 
Therefore, we decided to further investigate the structure of the H54A/E86A/G102K/H103A/N138G pentad mutant GAPR-1 (herein called the pentad mutant).

\subsection{The pentad mutation does not impact GAPR-1 tertiary structure}

We determined the $1.27 \AA$ A resolution X-ray crystal structure of the pentad mutant GAPR-1 (Fig. 5a). Pentad mutant GAPR-1 crystals belonged to space group $C 222_{1}$, with one molecule per asymmetric unit. WT GAPR-1 has previously been crystallized in the ligand-free state (Groves et al., 2004) and in the presence of $\mathrm{IP}_{6}$ (herein called $\mathrm{WTinIP}_{6}$ ), although $\mathrm{IP}_{6}$ was not visible in the crystal structure (van Galen et al., 2012). Like WT GAPR-1, the pentad mutant GAPR-1 has an $\alpha-\beta-\alpha$ sandwich fold (Fig. 5a) and residues packed in the core include a buried Glu65 that makes bidentate electrostatic interactions with Arg132 and hydrogen bonds to Ser55. The overall tertiary structure of pentad mutant GAPR-1 is very similar to those of WT and WTinIP $_{6}$ (Fig. 5a), with pairwise r.m.s.d.s of $1.72 \AA$ over $149 \mathrm{C}^{\alpha}$ atoms of WT GAPR-1 (PDB entry $1 \mathrm{smb}$ ) and $1.86 \AA$ over $149 \mathrm{C}^{\alpha}$ atoms of WTinIP $_{6}$ GAPR-1 (PDB entry 4aiw; van Galen et al., 2012). The GAPR-1 residues at positions 54, 86,102,103 and 138 have well defined electron density in both the pentad mutant (Supplementary Fig. S1a) and WT (Supplementary Fig. S1b) structures.

The interactions of the residues at positions 54, 86, 102, 103 and 138 are different in the WT and pentad mutant GAPR-1 monomers. In the pentad mutant GAPR-1 structure, Ala54, Ala86, Lys102, Ala103 and Gly138 are only involved in hydrophobic interactions (Fig. 5b). The Ala54 side chain packs against the Leu67 side chain. The Ala86 side chain packs against the side chains of Ala103 and Phe104. The aliphatic part of the Lys102 side chain packs against the aromatic ring of Phe97, while the charged amino group is solvent-exposed and does not make any interactions with the protein. In addition to packing with Ala86, the Ala103 side chain also interacts with the side chain of Trp83. Lastly, Gly138, which lacks a side chain, enables the packing of Phe97, Val140 and Phe145 against each other.

In contrast, the equivalent five residues in WT GAPR-1, His54, Glu86, Gly102, His103 and Asn138, also contribute polar interactions (Fig. 5c). The His54 imidazole $\mathrm{NH}$ forms an ionic bond to the Glu65 side-chain carboxylate $\mathrm{O}$ atom. One $\mathrm{O}$ atom of the Glu86 side-chain carboxylate forms ionic bonds to the His103 imidazole NH. In addition to the ionic bond to Glu86, the His103 imidazole NH forms a hydrogen bond to a water molecule. The His $103 \mathrm{C}^{\beta}$ atom also packs against the $\mathrm{C}^{\beta}$ atom of Trp83. Gly102 is not involved in any interactions within the subunit. Lastly, the Asn 138 side-chain carbonyl O atom forms a hydrogen bond to the Thr105 hydroxyl, while the aliphatic part of Asn138 packs against the aromatic ring of Phe97. Thus, the pentad mutations do not disrupt the tertiary structure, but do alter the interactions of residues within a monomer.

\subsection{The pentad mutation alters the GAPR-1 quaternary structure}

Like WT and WTinIP 6 GAPR-1, the pentad mutant GAPR-1 forms a crystallographic homodimer, with the subunits related by a twofold rotation about the $y$ axis. However, the dimer interface is significantly different in each of these three structures. Superposition of one subunit of the pentad mutant onto one subunit of the ligand-free WT GAPR-1 dimer reveals that the other subunit of pentad mutant GAPR-1 is rotated $38.1^{\circ}$ outwards relative to the corresponding ligandfree WT GAPR-1 monomer (Fig. 6a). In contrast, a similar superposition between pentad mutant and WTinIP $_{6}$ GAPR-1 shows a rotation of $17.9^{\circ}$ inwards relative to the other subunit of WTinIP 6 (Fig. 6b). Notably, neither the conserved residues at positions 54, 86, 102, 103 and 138 of WT GAPR-1 nor the mutated residues at equivalent positions in the pentad mutant GAPR-1 are directly involved in the dimer interface.

For the pentad mutant GAPR-1, the total surface area buried upon homodimerization is $945 \AA^{2}$, which constitutes

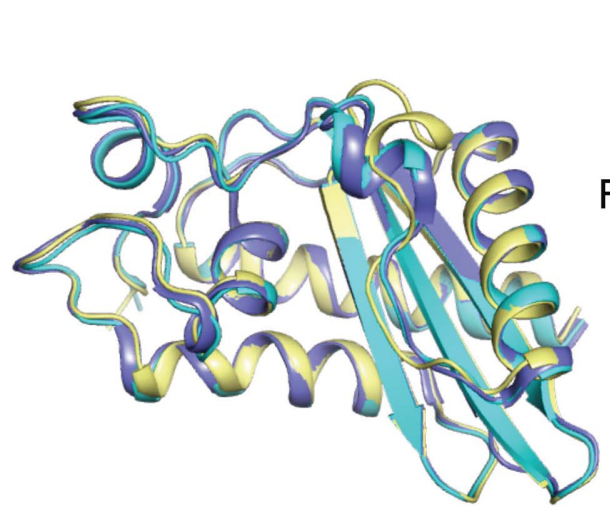

(a)

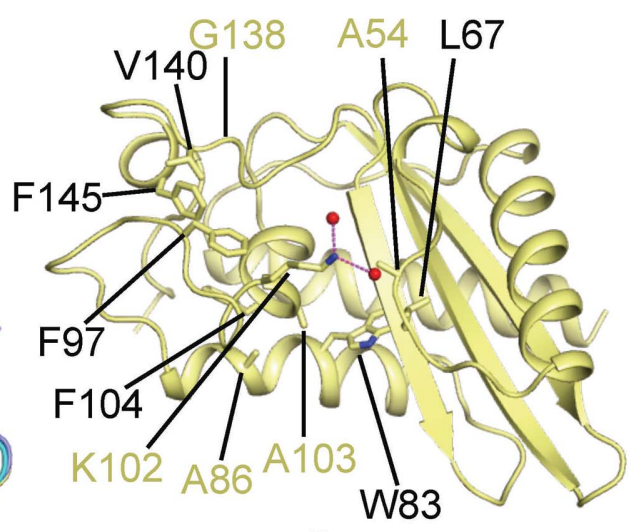

(b)

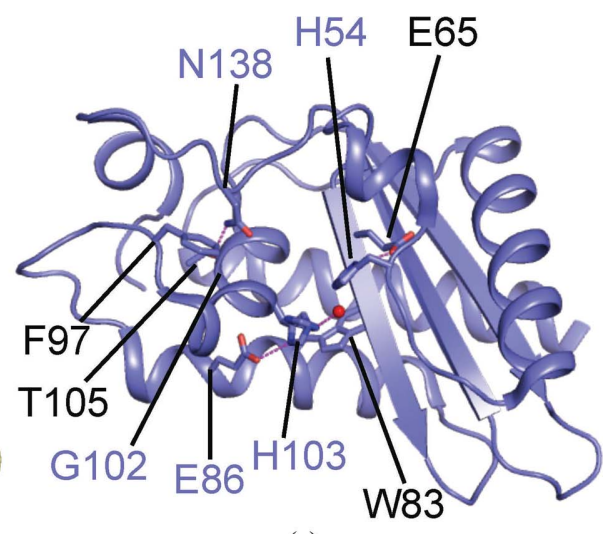

(c)

Figure 5

GAPR-1 tertiary structure is maintained in the pentad mutant. (a) Superimposed GAPR-1 monomers from different crystal structures: pentad mutant (yellow), ligand-free WT (violet) and WTinIP $_{6}$ (cyan). (b) Intramolecular interactions of the five mutated residues in pentad mutant GAPR-1. (c) Intramolecular interactions of the WT residues corresponding to those mutated in $(b)$. The side chains of mutated/WT residues and residues that interact with them in $(b)$ and $(c)$ are displayed in stick representation, with atoms colored by type: oxygen, red; nitrogen, blue; carbon, yellow (mutated) or violet (WT). Water molecules are displayed as red spheres. Mutated or WT residues are labeled in yellow or violet, respectively, and interacting residues are labeled in black. 
about $11.3 \%$ of the total surface area of each subunit. The dimer interface involves a total of 30 residues from each subunit and is stabilized by hydrophobic interactions and six intermolecular hydrogen bonds (Supplementary Fig. S2a). The three unique, inter-subunit hydrogen bonds are formed between the carbonyl O atoms of Leu52, Arg82 and Asp73, and the Arg50 guanidinium, the Tyr72 hydroxyl and the Lys88 amino group, respectively (Supplementary Fig. S2a). Further, we analysed the shape complementarity of the interacting surfaces in the crystallographic dimer, which quantifies the geometric surface complementarity $\left(S_{\mathrm{c}}\right)$ of protein-protein interfaces, where a score of 1.0 denotes perfect geometric complementarity of interacting interfaces (Lawrence \& Colman, 1993). Previous evaluation of protein-protein interaction interfaces identified and obtained by data mining the PDB showed that the $S_{\mathrm{c}}$ of crystallographically established interfaces clusters between 0.6 and 0.8 (De et al., 2005). The pentad mutant GAPR-1 dimer interface has an $S_{\mathrm{c}}$ of 0.70 , indicating that the geometrical complementarity at the pentad mutant dimer interface supports homodimer formation.

Similar to pentad mutant GAPR-1, the total surface area buried upon homodimerization of WT GAPR-1 is $943 \AA^{2}$, constituting approximately $11.7 \%$ of the total surface area of each subunit. The dimer interface involves a total of 26 residues from each subunit and is stabilized by two pairs of salt bridges, wherein Asp81 and Lys88 of one subunit form salt bridges with Lys88 and Asp81 of the other subunit, respectively (Supplementary Fig. S2b). Despite the similar total surface areas buried upon dimerization of the pentad mutant and WT GAPR-1, the WT GAPR-1 crystallographic dimer interface has an $S_{\mathrm{c}}$ of 0.60 , which is less than that of the pentad mutant, indicating that decreased shape complementarity of WT GAPR-1 is likely to adversely impact homodimerization.
The dimer interface is significantly less extensive for WTinIP $_{6}$. The total surface area buried upon dimerization is only $717 \AA^{2}$, constituting about $8.6 \%$ of the total surface area of each subunit. 23 residues from each subunit contribute to the interface and include a symmetry-related pair of hydrogen bonds. Each symmetry-related hydrogen bond is between the Leu52 carbonyl $\mathrm{O}$ atom of one subunit and the Arg50 mainchain amide of the other (Supplementary Fig. S2c). Unexpectedly, however, the WTinIP ${ }_{6}$ GAPR-1 dimer interface has an $S_{\mathrm{c}}$ of 0.69 , which is comparable to that of pentad mutant GAPR-1, but better than that of WT GAPR-1. Perhaps IP $_{6}$ induces conformational changes that improve the surface complementarity at the GAPR-1 homodimer interface.

Neither the five conserved/mutated residues, nor the residues that they interact with, are directly involved in the dimerinterface interactions (Figs. $5 b$ and $5 c$ ). However, a detailed examination of the structure provides clues to the stabilization of the different dimeric states. Although the WT and pentad mutant structures superimpose well, with a pairwise r.m.s.d. of $1.72 \AA$ over $149 \mathrm{C}^{\alpha}$ atoms, the C-terminal end of the $\alpha 4$ helix is distorted in pentad mutant GAPR-1 relative to the WT. This helix bears Glu86, one of the conserved/mutated residues, and Lys88, which is involved in the dimer interface. The WT GAPR-1 intramolecular Glu86-His103 interaction results in a $\mathrm{C}^{\alpha}$ distance of $7.3 \AA$, whereas the intramolecular interaction of the corresponding residues (Ala86 and Ala103) in pentad mutant GAPR-1 results in a $\mathrm{C}^{\alpha}$ distance of $6.1 \AA$. This distortion of the $\alpha 4$ helix is likely to cause the observed $1.5 \AA$ shift of the Lys $88 \mathrm{C}^{\alpha}$, and perhaps also causes the different rotomer adopted by the Lys 88 side chain. Therefore, although Lys 88 is involved in intermolecular ionic interactions across the dimer interfaces of both the WT and pentad mutant (Supplementary Figs. S2 $a$ and S2b), its altered conformation owing to the E86A and H103A mutations results in different intermolecular ionic interactions: with Asp81 in the WT GAPR-1 and Asp73 in the pentad mutant GAPR-1 dimer.

\subsection{The pentad mutation impacts the characteristics and accessibility of the equatorial groove}

Although the GAPR-1 mutations do not impact on the global tertiary structure, analysis of the surface electrostatic potential of the WT and the pentad mutant shows that the equatorial binding groove is more positively charged in pentad mutant GAPR-1 (Fig. 7) than in WT GAPR-1 (Supplementary Fig. S3). Further, the G102K mutation reduces the width and depth of the binding
Figure 6

The pentad mutation impacts GAPR-1 dimerization. Superimposition of the pentad mutant GAPR-1 dimer (gray and yellow subunits) onto either (a) ligand-free WT GAPR-1 (gray and violet subunits) or $(b)$ WTinIP6 GAPR-1 (gray and cyan subunits). The gray subunits of each dimer are superimposed. 
groove relative to the WT, as the lysine side chain occludes part of the binding groove in the mutant (Fig. 7 and Supplementary Fig. S3). The equatorial surface groove of the pentad mutant GAPR-1 has 15 ordered water molecules, of which two are hydrogen-bonded by the mutated Lys 102 side-chain amino group. The equatorial surface groove of WT GAPR-1 has 26 ordered water molecules, of which only one is hydrogenbonded by the His 103 imidazole NH. Therefore, the pentad mutations alter the arrangement and number of ordered water molecules bound to the GAPR-1 equatorial groove. Thus, Beclin 1 binding is also likely to be adversely impacted by the reduction in size, the change in shape and the increased positivity of the electrostatic surface of the equatorial binding groove in pentad mutant GAPR-1.
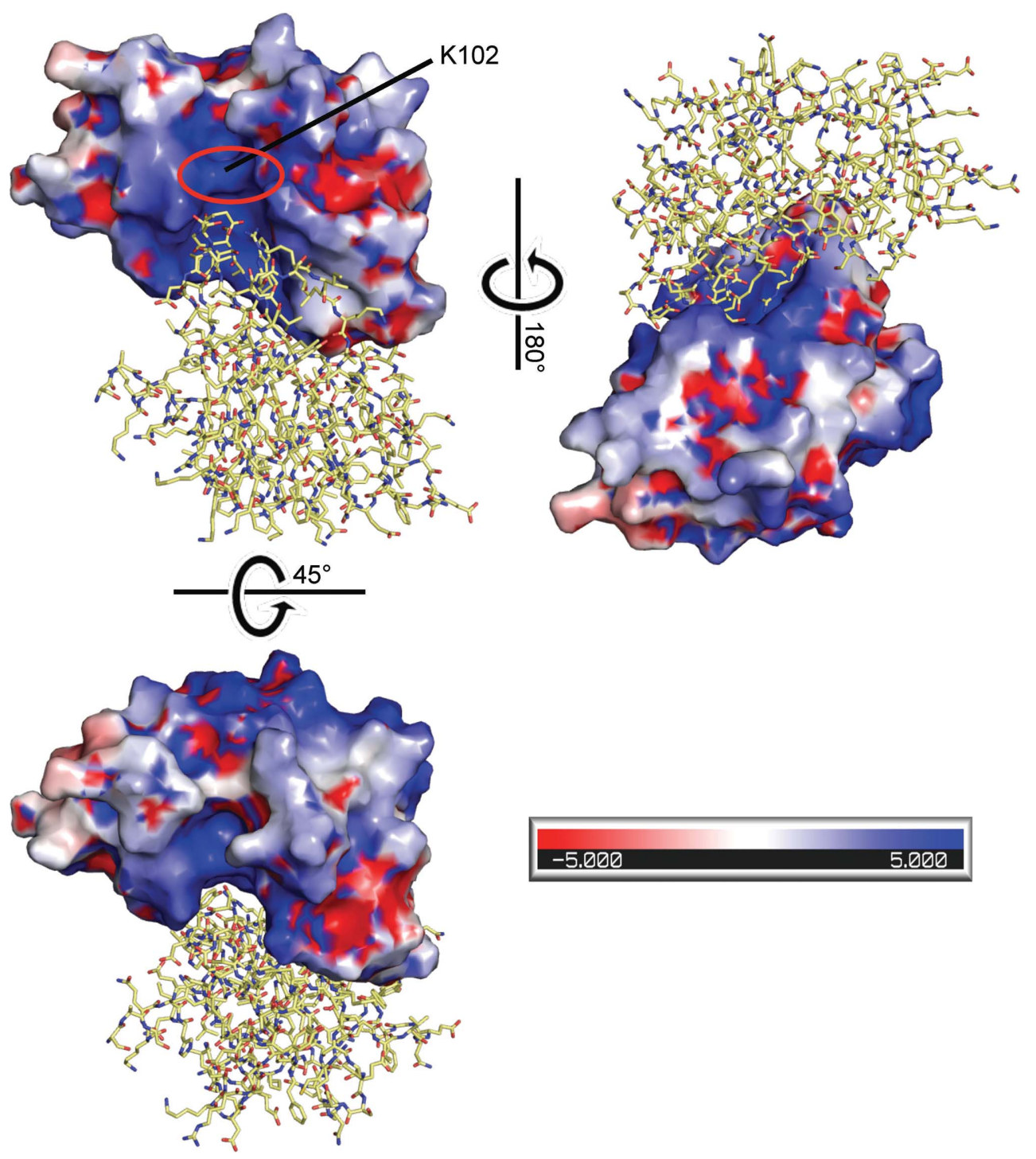

Figure 7

Pentad mutant GAPR-1 dimerization occludes the equatorial groove. The electrostatic potential surface of one subunit of the homodimer is shown, while the partner subunit is displayed in stick representation with atoms colored by atom type: oxygen, red; nitrogen, blue; carbon, yellow. The electrostatic potential surface of the same subunit of the homodimer is shown in the top left and bottom panels, while in the right panel the subunit is shown in stick representation, with the partner subunit shown as an electrostatic potential surface. Rotations representing the different views in each panel are indicated.
Strikingly, the equatorial groove of each subunit is substantially occluded by the other subunit upon dimerization of pentad mutant GAPR-1 (Fig. 7, left top and bottom panels), as well as in WT GAPR-1 (Supplementary Fig. S3, left top and bottom panels) and WTinIP 6 GAPR-1 (Supplementary Fig. S4, left top and bottom panels), with maximal occlusion of the groove in pentad mutant GAPR-1. Therefore, the binding of Beclin 1 is likely to depend on dissociation of the GAPR-1 dimer. An examination of the electrostatics of the interacting surfaces of pentad mutant GAPR-1 indicates that the more positive equatorial groove (Fig. 7, top left panel) interacts with a protrusion that bears negatively charged residues in the partner subunit (Fig. 7, top right panel). In contrast, in WT GAPR-1 the more negative equatorial groove (Supplementary Fig. S3, top left panel) interacts with a protrusion with fewer negative regions (Supplementary Fig. S3, top right panel), while in WTinIP $_{6}$ GAPR-1 the more neutral, equatorial groove (Supplementary Fig. S4, top left panel) interacts with a protrusion that is also more neutral (Supplementary Fig. S4, top right panel). Therefore, the altered electrostatic surface of the equatorial groove of the pentad mutant GAPR-1 appears to facilitate a stronger electrostatic interaction with an alternate surface on the partner GAPR-1 subunit, stabilizing the pentad mutant GAPR-1 dimer relative to the WT.

Given the increased shape and electrostatic complementarity of the dimer interface in the pentad mutant, it is plausible that the pentad mutant forms more stable dimers relative to the WT. The analysis of GAPR-1 dimerization in solution described below supports this hypothesis.

\subsection{The pentad mutation shifts the GAPR-1 monomer- dimer equilibrium towards dimerization}

The oligomeric state of WT GAPR-1 has not been unambiguously established; therefore, we first used analytical SEC to compare the size of WT and pentad mutant GAPR-1. In our hands, WT GAPR-1 elutes as a monomer for injected sample 
concentrations ranging from 0.25 to $2 \mathrm{~m} M$ (Supplementary Fig. S5a), with an estimated molecular mass of $17.7 \mathrm{kDa}$, which agrees well with the theoretical monomer molecular mass of 17.2 kDa calculated from the GAPR-1 sequence. Consistent with this, the Stokes radius $\left(R_{\mathrm{S}}\right)$ of $17 \AA$ estimated from analytical SEC of WT GAPR-1 is slightly smaller than the theoretical $R_{\mathrm{S}}$ of $19 \AA$ calculated for the WT GAPR-1 crystal monomer, and much smaller than the $R_{\mathrm{S}}$ of $24 \AA$ calculated for the WT GAPR-1 crystal dimer.

In contrast, the elution profiles of pentad mutant GAPR-1 are dependent on the concentration of sample injected onto the column (Supplementary Fig. S5b). When higher concentrations of 1 or $2 \mathrm{~m} M$ pentad mutant GAPR-1 are injected, the elution peaks are fairly symmetric, but are left-shifted relative to WT GAPR-1 (Supplementary Fig. S5c). The molecular mass estimated from these profiles is approximately $26 \mathrm{kDa}$, which is significantly larger than the $17.2 \mathrm{kDa}$ expected for a monomer, albeit smaller than the molecular mass of $34.4 \mathrm{kDa}$ expected for a dimer (Supplementary Fig. S5b). However, when lower concentrations of 0.5 or $0.25 \mathrm{~m} M$ pentad mutant GAPR-1 are injected, the elution peaks are right-shifted and are also slightly less symmetric than those at the higher concentrations, with the trailing edge of the $0.25 \mathrm{~m} M$ pentad mutant GAPR-1 elution peak overlapping the elution peak of WT GAPR-1 (Supplementary Fig. S5c), suggesting that the pentad mutant dimer dissociates as the concentration decreases and the elution profile includes both dimeric and monomeric species. The $R_{\mathrm{S}}$ of $21 \AA$ for pentad mutant GAPR-1 estimated from analytical SEC approximates the average of the theoretical $R_{\mathrm{S}}$ of the pentad mutant GAPR-1 crystal dimer $(24 \AA)$ and monomer $(19 \AA)$.

ITC was used to quantify the self-dissociation constants of WT and pentad mutant GAPR-1. We do not detect any dimer dissociation for WT GAPR-1 (Supplementary Fig. S6a). While hypothetically this may be the result of factors such as extremely tight binding or heats of dissociation that are beyond the detection range of this method, the most likely explanation, which is consistent with the SEC analysis reported above, is that WT GAPR-1 does not dimerize at these concentrations. In contrast, the pentad mutant GAPR-1 homodimerizes with an apparent $K_{\mathrm{d}}$ of $29 \pm 7 \mu M$ (Supple- mentary Fig. S6b). Further, dimerization of pentad mutant GAPR-1 is favored by both entropy $(\Delta S=20 \pm$ $\left.33 \mathrm{~J} \mathrm{~K}^{-1} \mathrm{~mol}^{-1}\right)$ and enthalpy $\left(\Delta H=-19 \pm 9 \mathrm{~kJ} \mathrm{~mol}^{-1}\right)$. Notably, the injection of $1 \mathrm{~m} M$ pentad mutant GAPR-1 onto a SEC column results in elution concentrations varying between 2 and $41 \mu M$ (Supplementary Table S1). The leading edge of the SEC elution peak has concentrations above (Supplementary Table S1) the dimer dissociation constant quantified by ITC. However, the concentration of fractions that correspond to the trailing part of the peak range between 2 and $23 \mu M$, which is lower than the $K_{\mathrm{d}}$ and may result in dissociation of the dimer. Therefore, the ITC results verify the improved homodimerization of pentad mutant GAPR-1 relative to WT GAPR-1, which agrees with the increased shape and electrostatic complementarity of the dimer interface of the pentad mutant relative to WT, as well as the analytical SEC results indicating that WT GAPR-1 is a monomer in solution while the pentad mutant GAPR-1 favors a dimer. Lastly, in order to confirm the oligomeric state of WT and pentad mutant GAPR-1, we analysed homogeneous samples of each protein by SEC-SAXS.

\subsection{SEC-SAXS confirms that WT GAPR-1 is a monomer in solution}

$I(0)$ varies across the SEC-SAXS peak of WT GAPR-1 (Supplementary Fig. S7a), consistent with the variation in elution concentration (Supplementary Table S1). The $R_{\mathrm{g}}$ determined from intensity-normalized, buffer-subtracted data is constant across the scattering peak of WT GAPR-1 (Fig. 8a), indicating that the $R_{\mathrm{g}}$ is not dependent on the WT GAPR-1 concentration in the concentration range tested. SVD analysis was used to determine the minimum number of species required to represent the whole scattering peak. SVD analysis of a matrix containing buffer and WT GAPR-1 scattering data normalized by the incident beam intensity indicates that the sample contained only one dominant species besides the buffer, based on the magnitude of the singular values, autocorrelations of the columns of $U$ and $V$ (Fig. $8 b$ ), and plots of columns of $U$ (Fig. 8c).

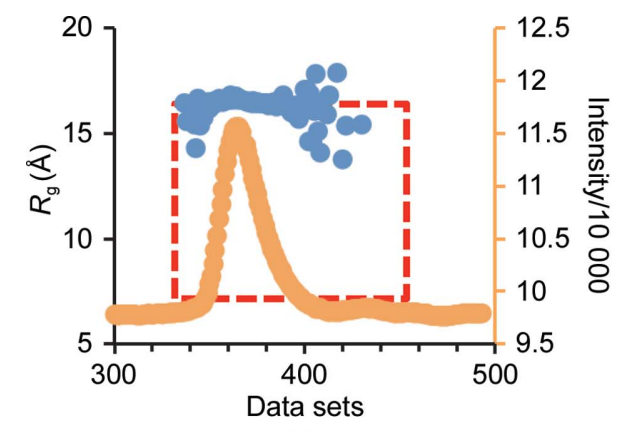

(a)

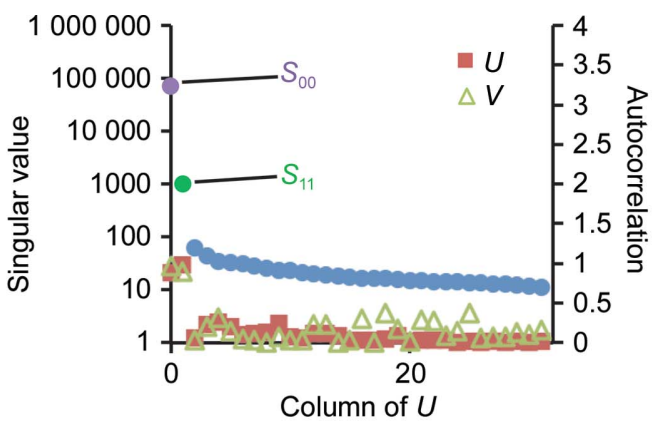

(b)

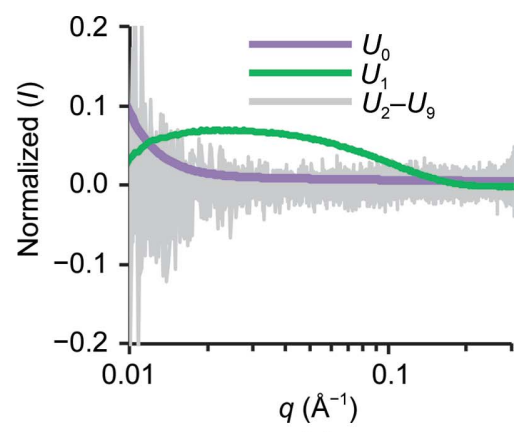

(c)

Figure 8

WT GAPR-1 SEC-SAXS data showing the $R_{\mathrm{g}}$ distribution across the scattering peak and SVD analysis. $(a)$ Raw scattering data (orange) and $R_{\mathrm{g}}$ distribution (blue). (b) Singular values (primary $y$ axis, shown as dots) and autocorrelations of the columns of $U$ (secondary $y$ axis, shown as red squares) and $V$ (secondary $y$ axis, shown as green triangles) of the data from $(a)$. (c) Columns of the orthonormal $U$ matrix multiplied by the corresponding rankordered singular values in $(b)$. 
Ten data sets with similar $R_{\mathrm{g}}$ were selected, scaled and averaged for further data analysis. The linear Guinier plots at $q R_{\mathrm{g}}<1.3$ indicate that the sample is monodisperse (Fig. 9a). The $R_{\mathrm{g}}$ of $16 \AA$ estimated from the Guinier plot agrees well with the $R_{\mathrm{g}}$ of $15 \AA$ estimated from the $P(r)$ curve (Fig. $9 b$ ) and the $R_{\mathrm{g}}$ of $15 \AA$ calculated from the WT GAPR- 1 crystal structure monomer, but is substantially smaller than the $R_{\mathrm{g}}$ of $20 \AA$ calculated from the WT GAPR-1 crystal structure dimer. The $P(r)$ curve indicates that WT GAPR-1 is globular, with a maximum molecular dimension $\left(D_{\max }\right)$ of $58 \AA$ (Fig. $9 b$ ). The dimensionless Kratky plot suggests that WT GAPR-1 has limited disorder (Fig. 9c). The normalized spatial discrepancy of the ten generated $a b$ initio molecular models is $0.46 \pm 0.02$. The envelope confirms the globular shape of WT GAPR-1 (Fig. 9d). Further, the molecular mass estimated from the volume of correlation, $V_{\mathrm{c}}$ (Rambo \& Tainer, 2013), is $14.6 \mathrm{kDa}$, which is a little smaller than the theoretical monomeric molecular mass of $17.2 \mathrm{kDa}$.

Theoretical scattering curves calculated from either the WT GAPR-1 monomer or dimer were compared with the

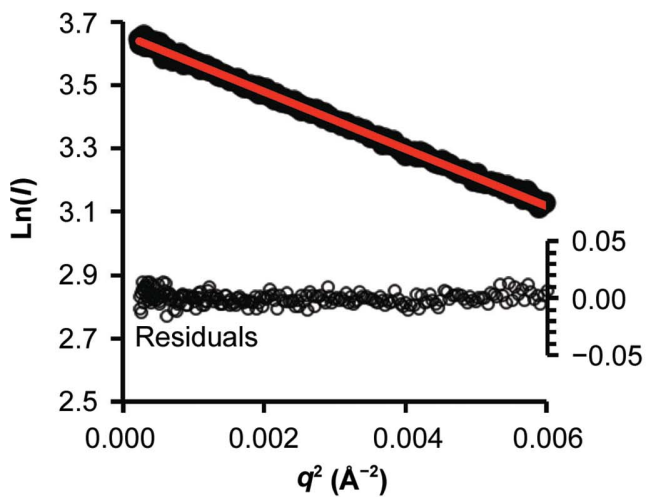

(a)
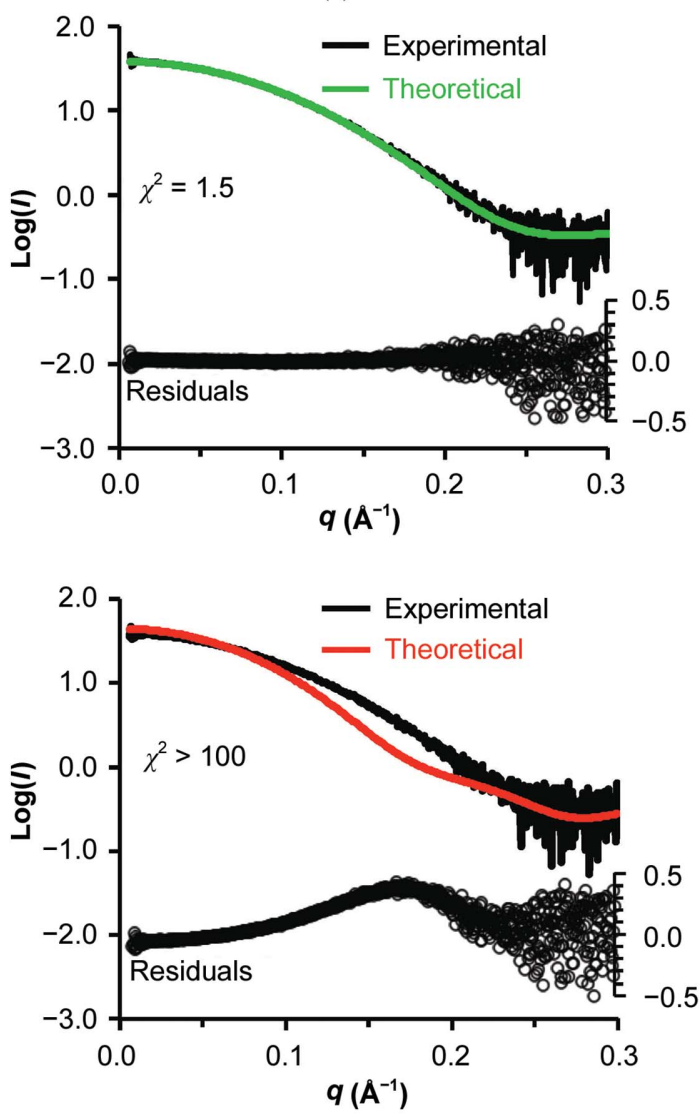

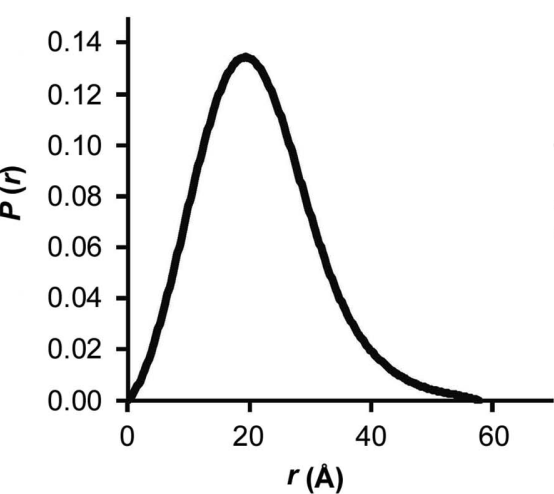

(b)
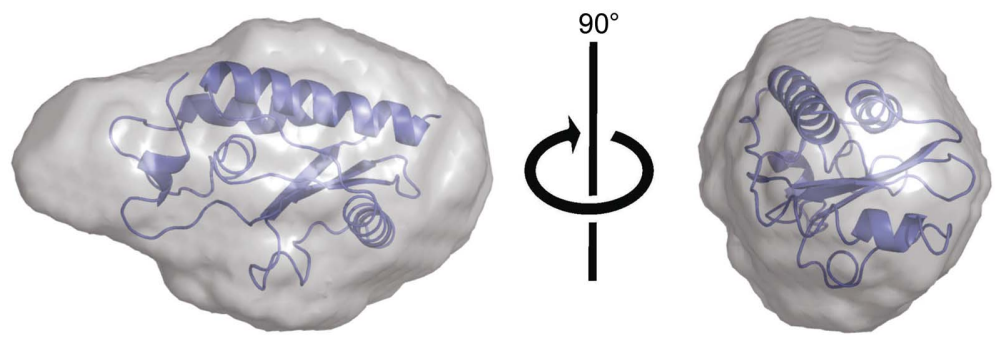

$(d)$
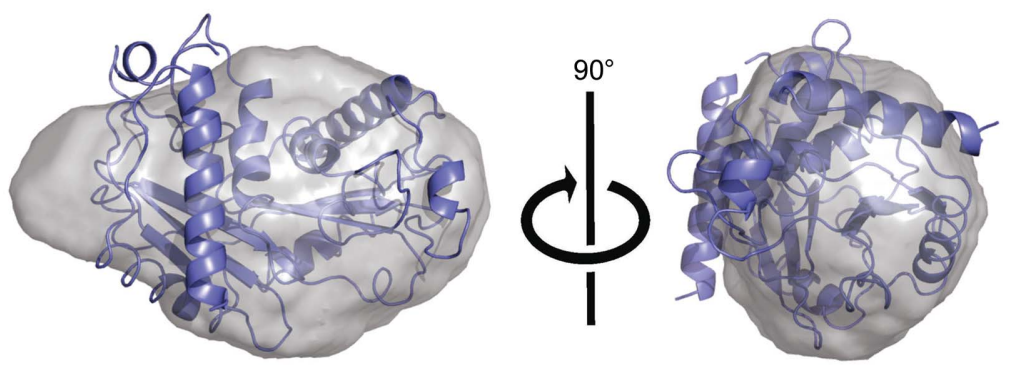

$(e)$

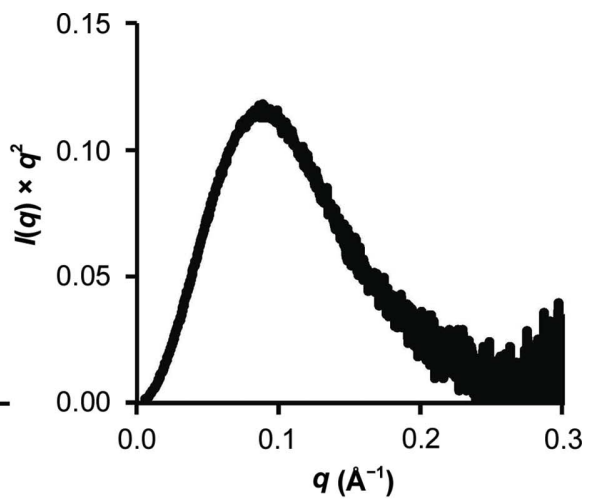

$(c)$

Figure 9

SAXS analysis of WT GAPR-1. (a) Guinier plot. (b) $P(r)$ distribution. (c) Kratky plot. (d) Fit of the WT crystal structure monomer to the SAXS data. Left panel: the theoretical scattering curve calculated from the WT crystal structure monomer (green) fitted to experimental scattering data (black). Right panel: WT monomer (violet ribbon) docked into the SAXS molecular envelope (gray surface) shown in two orthogonal orientations. (e) Fit of the WT dimer crystal structure to the SAXS data. Left panel: the theoretical scattering curve calculated from the WT dimer crystal structure (red) fitted to the experimental scattering data (black). Right panel: WT dimer (violet ribbon) docked into the SAXS envelope (gray surface) shown in two orthogonal orientations. 
experimental scattering data (Figs. $9 d$ and $9 e$ ). The theoretical scattering curve calculated from the monomer agreed well with the experimental scattering curve, as indicated by a $\chi^{2}$ of 1.5 (Fig. $9 d$, left panel), while the theoretical curve calculated from the dimer fitted very poorly, as indicated by a $\chi^{2}$ larger than 100 (Fig. $9 e$, left panel). The deviation of $\chi^{2}$ from a perfect value of 1.0 may be because the WT GAPR- 1 crystal structure lacks coordinates for the linker (GPLGS) preceding GAPR-1, as well as for residues 1-3 and 153-154. This good fit indicated by the $\chi^{2}$ is consistent with our visual observation that the SAXS envelope fits the monomeric WT GAPR-1 crystal structure well (Fig. $9 d$, right panel) but is too small to accomodate the WT GAPR-1 dimer (Fig. 9e, right panel). Therefore, our SEC-SAXS and ITC data consistently confirm that WT GAPR-1 is a monomer in solution at the concentrations tested.

\subsection{The pentad mutant GAPR-1 transitions between} monomeric and dimeric states in solution

Similar to WT GAPR-1, $I(0)$ also varies across the SECSAX peak of pentad mutant GAPR-1 (Supplementary Fig. $\mathrm{S} 7 b$ ), consistent with the variation in elution concentration (Supplementary Table S1). However, in contrast to WT GAPR-1, the $R_{\mathrm{g}}$ distribution determined from the intensitynormalized, buffer-subtracted SEC-SAXS data of pentad mutant GAPR-1 is inconsistent across the scattering peak (Fig. 10). The $R_{\mathrm{g}}$ calculated from SAXS data sets at the leading part of the peak is $\sim 20 \AA$ (here called the $\sim 20 \AA R_{\mathrm{g}}$ peak part), while the $R_{\mathrm{g}}$ calculated from SAXS data sets at the trailing part of the peak is $\sim 16 \AA$ (here called the $\sim 16 \AA R_{\mathrm{g}}$

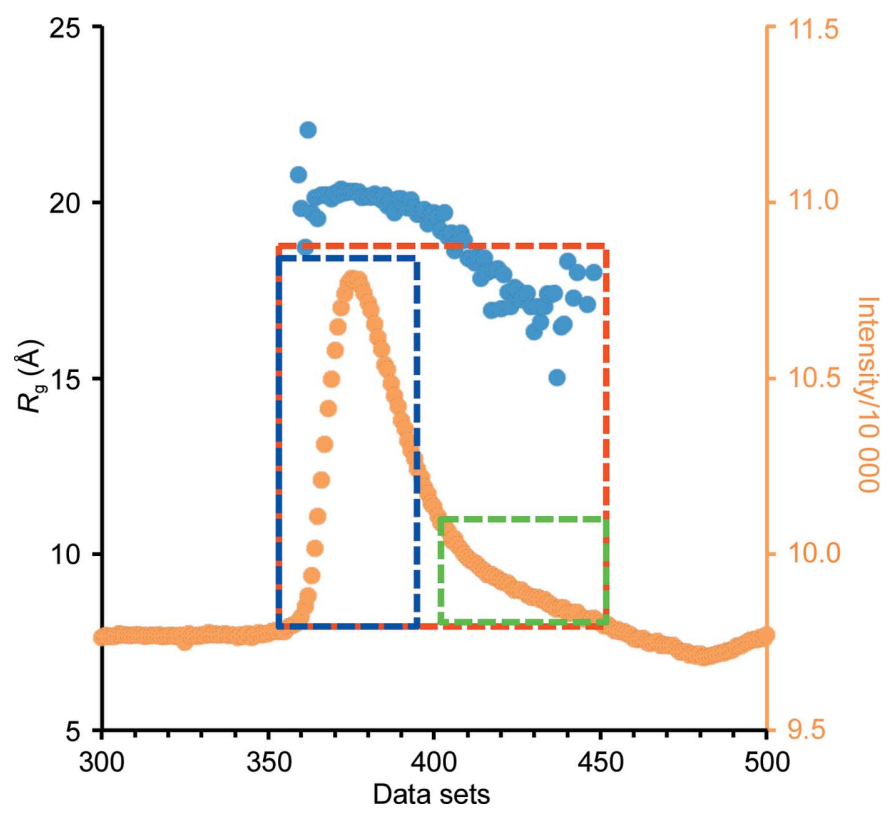

Figure 10

$R_{\mathrm{g}}$ distribution across the SEC-SAXS elution peak. Raw scattering data (orange) and $R_{\mathrm{g}}$ distribution (blue) with data selected for SVD analysis of the whole peak (red box), the leading part of the peak with an $R_{\mathrm{g}}$ of $\sim 20 \AA$ (blue box) and the trailing part of the peak with an $R_{\mathrm{g}}$ of $\sim 16 \AA$ (green box). peak part), with intervening SAXS data sets having intermediate $R_{\mathrm{g}}$ values between 16 and $20 \AA$. The minimum $R_{\mathrm{g}}$ estimated from the pentad mutant GAPR-1 SAXS data is comparable to that calculated from a monomer of the pentad mutant crystal structure and is also similar to that observed for WT GAPR-1. The maximum $R_{\mathrm{g}}$ estimated from the pentad mutant GAPR-1 SAXS data is similar to the $R_{\mathrm{g}}$ of $20 \AA$ calculated from the pentad mutant GAPR-1 dimer in the crystal structure. Together, these data indicate that at the concentrations used for these SEC-SAXS experiments the pentad mutant GAPR-1 may transition between dimeric and monomeric states.

SVD analysis was also performed on SAXS of the pentad mutant GAPR-1 to determine the minimum number of species required to represent either the whole scattering peak (red box in Fig. 10; Figs. 11a and 11b), the $\sim 20 \AA R_{\mathrm{g}}$ peak part (blue box in Fig. 10; Figs. $11 c$ and $11 d$ ) and the $\sim 16 \AA R_{\mathrm{g}}$ peak part (green box in Fig. 10; Figs. 11e and 11f). Contrary to our expectations based on the observed decrease in $R_{\mathrm{g}}$ across the scattering peak, SVD analysis of the whole scattering peak (Fig. 11a) indicates that in addition to the buffer, $S_{00}$, the sample contains only one significant component, $S_{11}$, as the next nonbuffer species, $S_{22}$, cannot be considered significant based on the autocorrelations of the columns of $U$ and $V$ (Fig. 11a) and the plot of the columns of $U$ (Fig. 11b). Not surprisingly, selection of SAXS data sets from only the $\sim 20 \AA$ $R_{\mathrm{g}}$ peak part (blue box in Fig. 10; Figs. $11 c$ and $11 d$ ) also indicates the presence of only one species besides the buffer. Likewise, selection of SAXS data sets from the $\sim 16 \AA R_{\mathrm{g}}$ peak part (green box in Fig. 10; Figs. 11e and 11f) also identified only one nonbuffer component, $S_{11}$. Notably, however, this $S_{11}$ species has a singular value of $\sim 100$ (Fig. 11c), which is substantially less than the magnitude of the singular value of the $S_{11}$ species identified in the analysis of the $\sim 20 \AA R_{\mathrm{g}}$ peak part (Fig. 11b), suggesting that the concentration of the nonbuffer component is much lower in the $\sim 16 \AA R_{\mathrm{g}}$ peak part relative to that in the $\sim 20 \AA R_{\mathrm{g}}$ peak part. Further, the magnitude of the singular value of the $S_{11}$ species in the $\sim 16 \AA$ $R_{\mathrm{g}}$ peak part is comparable to that of the insignificant $S_{22}$ species in the SVD analysis of the whole peak, raising the possibility that a third species may be present at the trailing part but at concentrations that are too low for identification as a distinct significant species in the analysis of the whole peak. Another possibility is that the $S_{11}$ species in the $\sim 16 \AA R_{\mathrm{g}}$ peak part simply corresponds to a low concentration of the $S_{11}$ species in the $\sim 20 \AA R_{\mathrm{g}}$ peak part; however, this possibility does not explain the decrease in $R_{\mathrm{g}}$ observed at the trailing part. To further characterize the size and shape of the significant species in the two parts of the scattering peak, ten buffersubtracted data sets were selected from either the $\sim 20 \AA$ $R_{\mathrm{g}}$ peak part or from the $\sim 16 \AA R_{\mathrm{g}}$ peak part, which were separately scaled and averaged for subsequent data analysis.

For the averaged pentad mutant GAPR-1 SAXS data derived from the $\sim 20 \AA R_{\mathrm{g}}$ peak part, the Guinier plot is linear at $q R_{\mathrm{g}}<1.3$, indicating that this eluted sample was monodisperse (Fig. 12a). The $R_{\mathrm{g}}$ estimated from Guinier plot 
analysis and $P(r)$ analysis is $20 \AA$ (Figs. $12 a$ and $12 b$ ), which is substantially larger than the $R_{\mathrm{g}}$ of $16 \AA$ estimated for WT GAPR-1, as well as the $R_{\mathrm{g}}$ of $15 \AA$ calculated from the pentad mutant GAPR-1 crystal structure monomer, but comparable to the $R_{\mathrm{g}}$ of $20 \AA$ A calculated from the pentad mutant GAPR-1 crystal structure dimer. The $P(r)$ plot indicates that the pentad mutant GAPR-1 in the $\sim 20 \AA$ peak part is globular, with a $D_{\max }$ of $66 \AA$ (Fig. 12b), which is larger than the $D_{\max }$ of $58 \AA$ estimated from the WT GAPR-1 SAXS data, as well as the $D_{\max }$ of $56 \AA$ calculated from the pentad mutant crystal structure monomer, but smaller than the $D_{\max }$ of $72 \AA$ calculated from the pentad mutant GAPR-1 crystal structure dimer. The Kratky plot indicates that pentad mutant GAPR-1 is also well folded (Fig. 12c). The molecular mass of pentad mutant GAPR-1 estimated from the $V_{\mathrm{c}}$ is $24.6 \mathrm{kDa}$, which is comparable to the mass of $26 \mathrm{kDa}$ estimated from analytical SEC, but smaller than the theoretical molecular mass of $34.4 \mathrm{kDa}$ calculated for a dimer and larger than the theoretical molecular mass of $17.2 \mathrm{kDa}$ for a monomer. The normalized spatial discrepancy of the ten generated ab initio molecular models calculated from these data is $0.58 \pm 0.02$. Notably, the theoretical scattering curve calculated from the pentad mutant GAPR-1 crystal structure monomer does not fit the experimental scattering curve, as indicated by the $\chi^{2}$ of 96 (Fig. 12d, left panel), but the theoretical scattering curve calculated from the pentad mutant GAPR-1 crystal structure dimer agrees with the experimental scattering curve, as indicated by a $\chi^{2}$ of 2.2 (Fig. 12e, left panel). Similar to the WT GAPR-1 crystal structure, the pentad mutant GAPR-1 crystal structure used to calculate the theoretical scattering curves lacks coordinates owing to missing electron density for linker residues GPLGS and GAPR-1 residues 1-3, but not for GAPR-1 residues 153154 (Table 1). Therefore, it is unlikely that these missing residues account for the relatively elevated $\chi^{2}$ of 2.2 ; rather, this is probably indicative of an imperfect fit. Visual observation indicates that the SAXS envelope is much larger than a pentad mutant monomer (Fig. 12d, right panel), but fits the pentad mutant dimer structure well (Fig. 12e, right panel). Measurement of SEC eluate concentration indicates a range of 31-41 $\mu M$ across the $\sim 20 \AA R_{\mathrm{g}}$ peak part, which is slightly above the apparent dimer dissociation $K_{\mathrm{d}}(29 \pm 7 \mu M)$ (Supplementary Table S1). Thus, our data indicate that the pentad mutant GAPR-1 eluting under the $\sim 20 \AA R_{\mathrm{g}}$ peak part is in a dimeric state.

For the averaged pentad mutant GAPR-1 SAXS data derived from the $\sim 16 \AA R_{\mathrm{g}}$ peak part, the Guinier plot is linear at $q R_{\mathrm{g}}<1.3$, indicating that this eluted sample was also monodisperse (Fig. 13a). The $R_{\mathrm{g}}$ of $17 \AA$ A estimated from Guinier

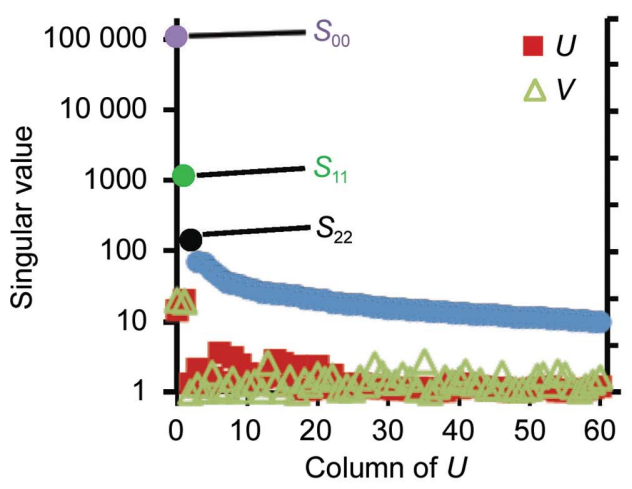

(a)

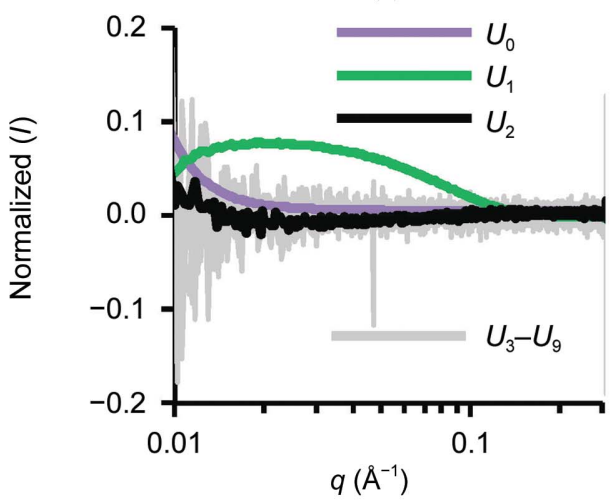

(b)

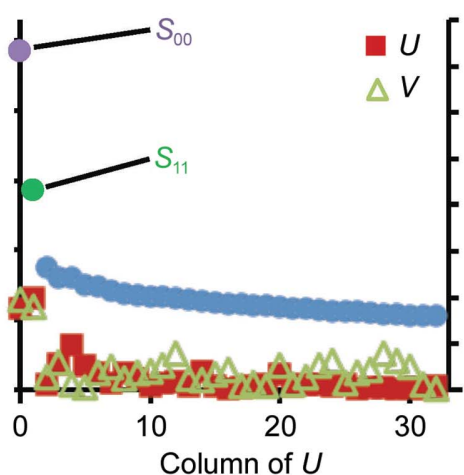

(c)

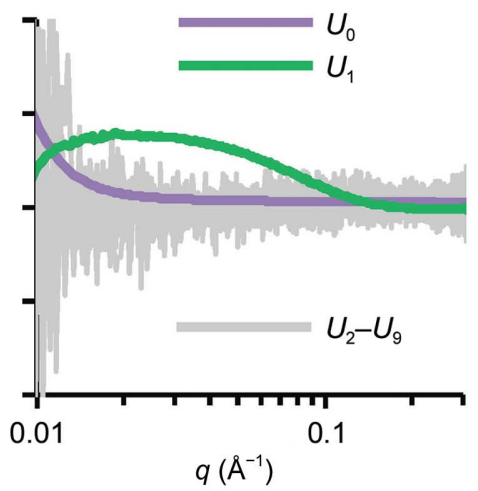

(d)

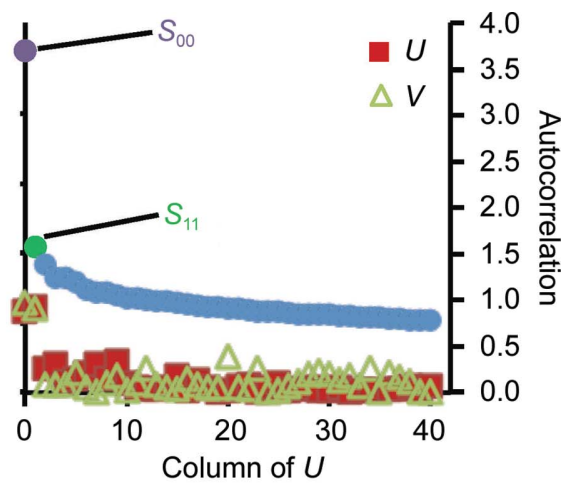

(e)

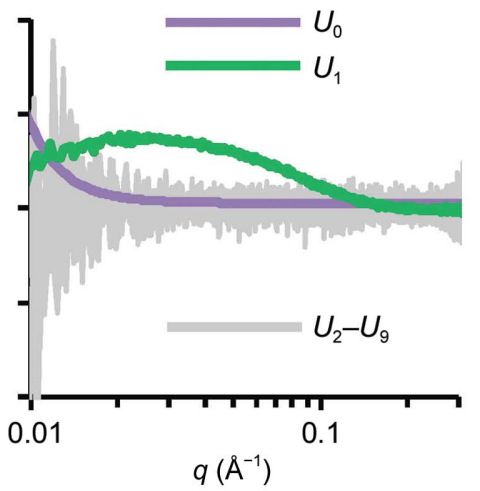

$(f)$

Figure 11

SVD analysis of SEC-SAXS from pentad mutant GAPR-1. (a) Singular values (primary $y$ axis, shown as dots) and autocorrelations of the columns of $U$ (secondary $y$ axis, shown as red squares) and $V$ (secondary $y$ axis, shown as red squares) of the data encompassing the whole peak indicated by the red box in Fig. 10. (b) Columns of the orthonormal $U$ matrix multiplied by the corresponding rank-ordered singular values in $(a)$. (c) Singular values and autocorrelations of the columns of $U$ and $V$ of the data encompassing the leading edge of the peak indicated by the blue box in Fig. 10. $(d)$ Columns of the orthonormal $U$ matrix multiplied by the corresponding rank-ordered singular values in (c). (e) Singular values and autocorrelations of the columns of $U$ and $V$ of the data encompassing the trailing edge of the peak indicated by the green box in Fig. 10. $(f)$ Columns of the orthonormal $U$ matrix multiplied by the corresponding rank-ordered singular values in $(e)$. 
and $P(r)$ analysis (Figs. $13 a$ and $13 b$ ) is similar to the $R_{\mathrm{g}}$ of $16 \AA$ estimated for the WT GAPR-1 SAXS data and is slightly larger than the $R_{\mathrm{g}}$ of $15 \AA$ calculated from the pentad mutant GAPR-1 crystal structure monomer, but is significantly smaller than the $R_{\mathrm{g}}$ of $20 \AA$ calculated from the pentad mutant GAPR-1 crystal structure dimer. The $P(r)$ plot indicates that particles in the $\sim 16 \AA R_{\mathrm{g}}$ peak part are globular, with a $D_{\text {max }}$ of $56 \AA$ (Fig. 13b), which is similar to the $D_{\max }$ of WT
GAPR-1. The Kratky plot indicates that these particles are also well folded (Fig. 13c). The molecular mass estimated from the $V_{\mathrm{c}}$ is $21 \mathrm{kDa}$, which is larger than the theoretical molecular mass of $17.2 \mathrm{kDa}$ for monomeric pentad mutant GAPR-1, but much smaller than $34.4 \mathrm{kDa}$, the theoretical molecular mass of dimeric pentad mutant GAPR-1. The normalized spatial discrepancy of the ten generated ab initio molecular models is $0.50 \pm 0.05$. Lastly, the theoretical scattering curve calculated

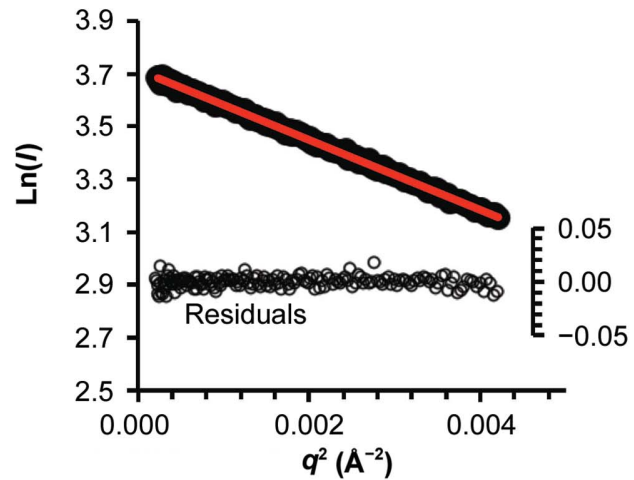

(a)
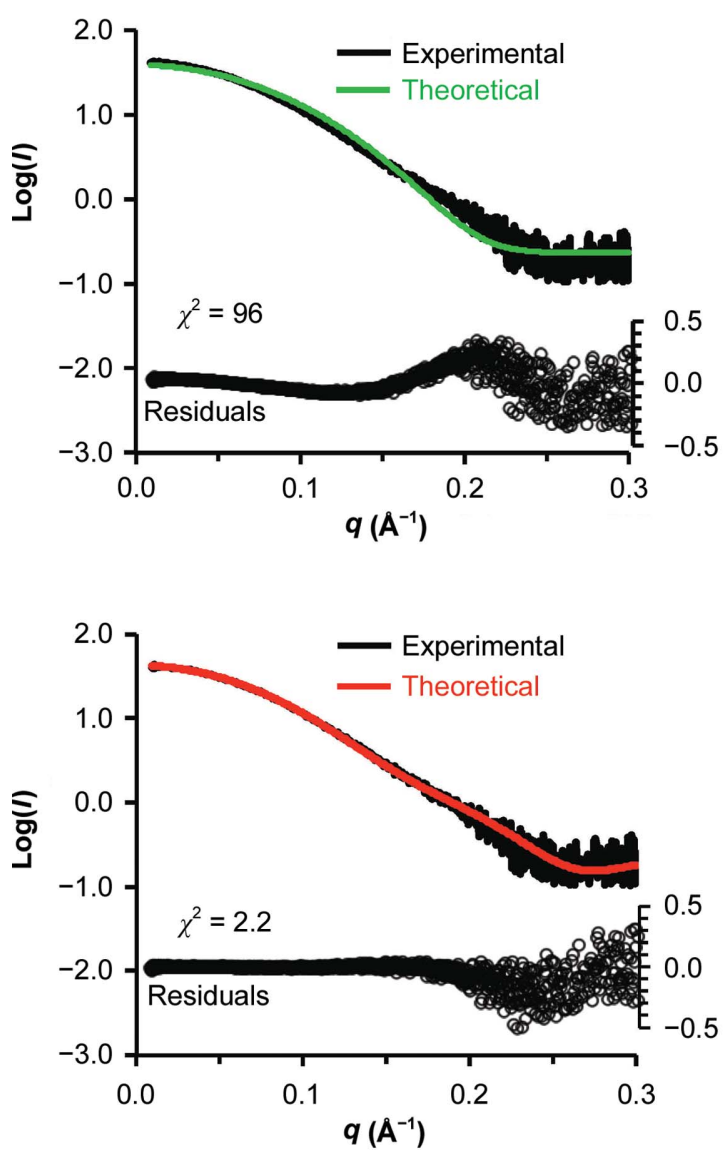

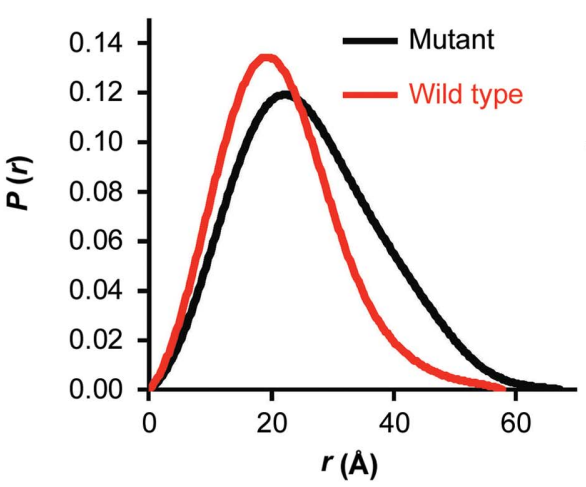

(b)

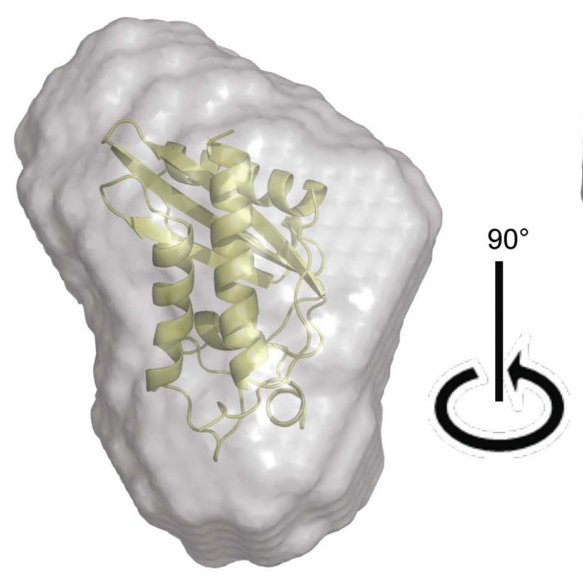

(d)

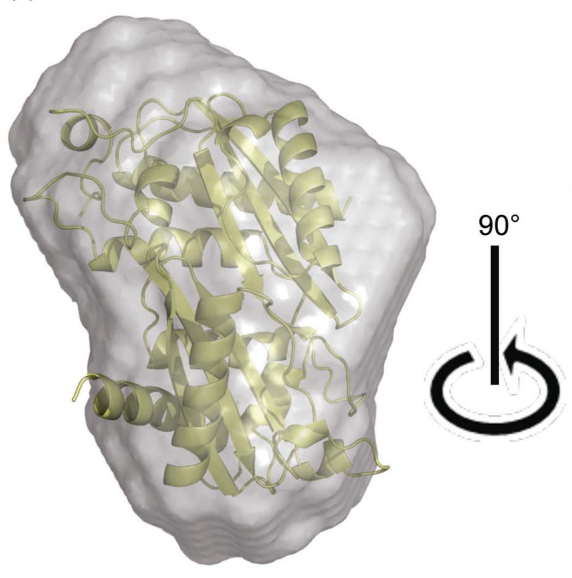

$(e)$

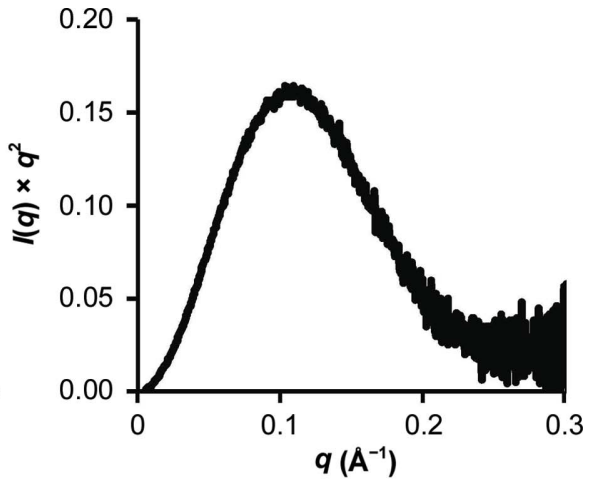

$(c)$
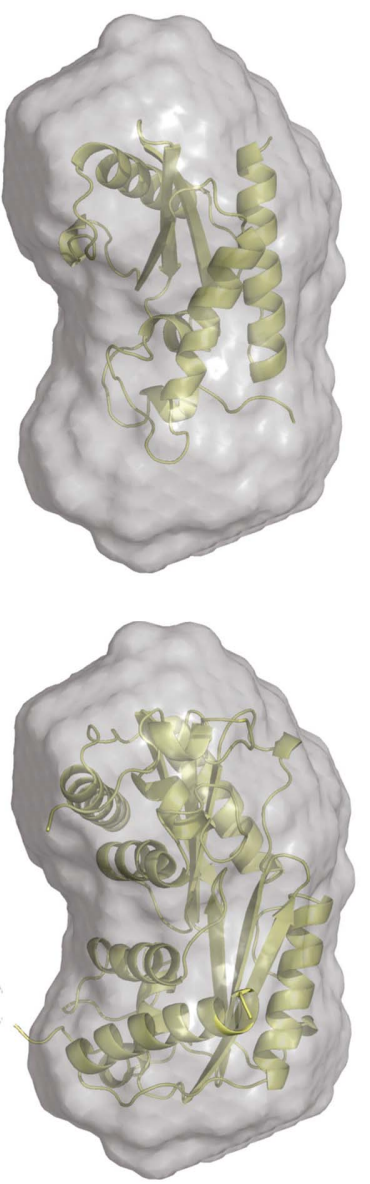

Figure 12

Analysis of the $\sim 20 \AA R_{\mathrm{g}}$ peak part of the pentad mutant GAPR-1 SAXS. (a) Guinier plot. $(b) P(r)$ distribution. $(c)$ Kratky plot. $(d)$ Fit of the pentad mutant crystal structure monomer to the SAXS data. Left panel: the theoretical scattering curve calculated from the pentad mutant monomer (green) fitted to the experimental scattering data (black). Right panel: the pentad mutant crystal structure monomer (yellow ribbon) docked into the SAXS molecular envelope (gray surface) shown in two orthogonal orientations. (e) Fit of the pentad mutant crystal structure dimer to the SAXS data. Left panel: the theoretical scattering curve calculated from the pentad mutant monomer (red) fitted to the experimental scattering data (black). Right panel: the pentad mutant crystal structure dimer (yellow ribbon) fitted to the SAXS molecular envelope (gray surface) shown in two orthogonal orientations. 


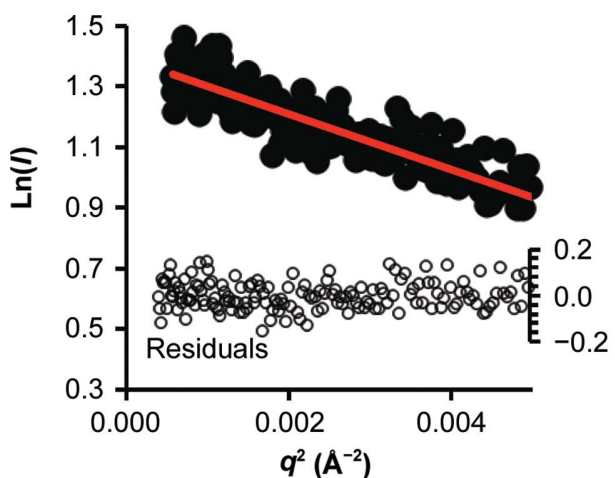

(a)
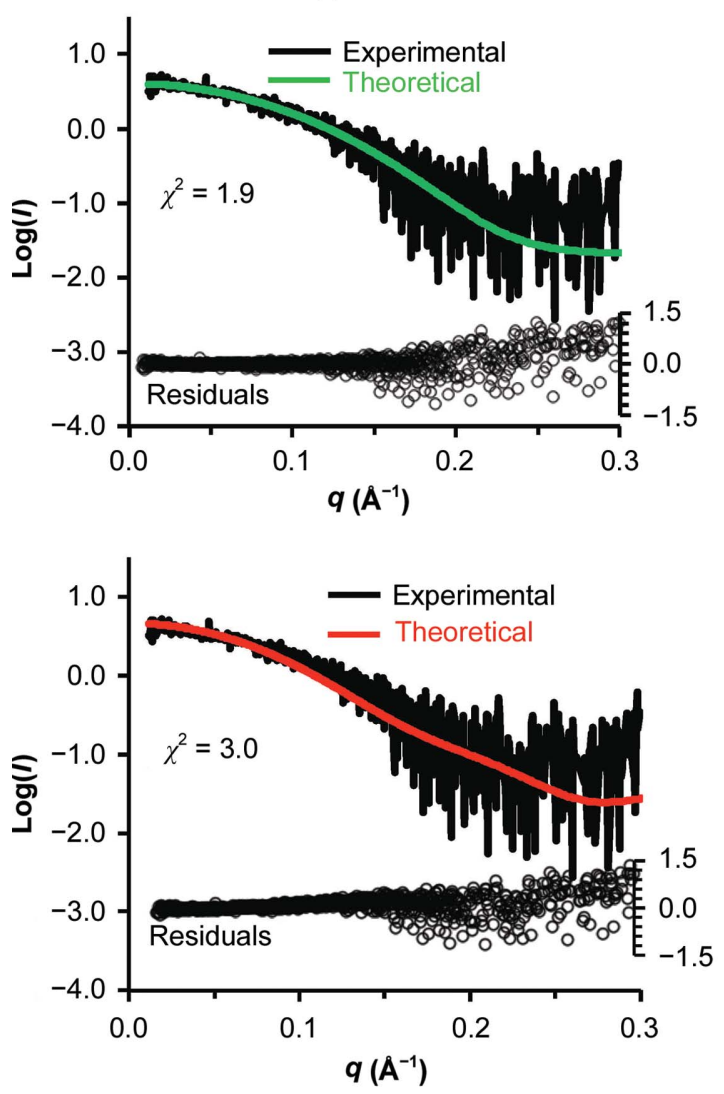

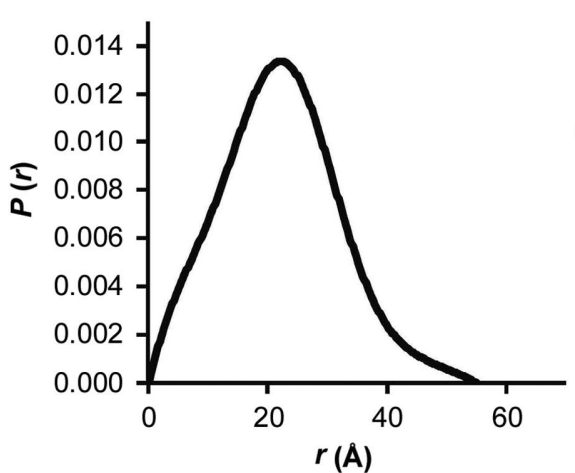

(b)

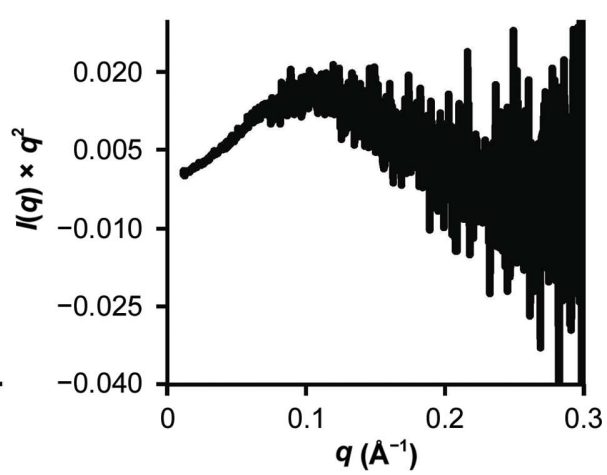

(c)

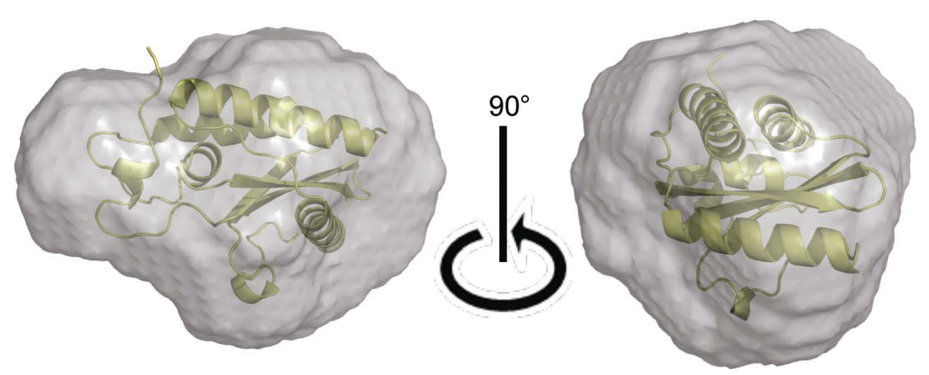

(d)

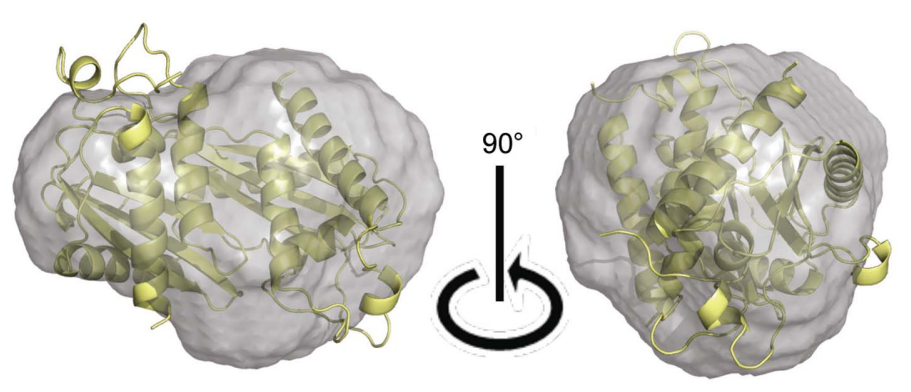

$(e)$

Figure 13

Analysis of the $\sim 16 \AA R_{\mathrm{g}}$ peak part of the pentad mutant GAPR-1 SAXS. (a) Guinier plot. $(b) P(r)$ distribution. $(c)$ Kratky plot. $(d)$ Fit of the pentad mutant crystal structure monomer to the SAXS data. Left panel: the theoretical scattering curve calculated from the pentad mutant monomer (green) fitted to the experimental scattering data (black). Right panel: the pentad mutant crystal structure monomer (yellow ribbon) docked to the SAXS molecular envelope (gray surface) shown in two orthogonal orientations. (e) Fit of the pentad mutant crystal structure dimer to the SAXS data. Left panel: the theoretical scattering curve calculated from the pentad mutant monomer (red) fitted to the experimental scattering data (black). Right panel: the pentad mutant crystal structure dimer (yellow ribbon) docked to the SAXS molecular envelope (gray surface) shown in two orthogonal orientations.

from the pentad mutant GAPR-1 crystal structure monomer fitted the experimental scattering curve well with a $\chi^{2}$ of 1.9 (Fig. 13d, left panel), somewhat better than the fit indicated by a $\chi^{2}$ of 3.0 for the theoretical scattering curve calculated from the pentad mutant GAPR-1 crystal structure dimer (Fig. 13e, left panel). Visual observation indicates that a pentad mutant monomer fits the SAXS envelope calculated from the $\sim 16 \AA$ $R_{\mathrm{g}}$ peak part SAXS data better (Fig. 13d, right panel) than the pentad mutant dimer structure (Fig. 13e, right panel). Thus, all of our data suggest that the pentad mutant GAPR-1 eluting under the $\sim 16 \AA R_{\mathrm{g}}$ peak part is likely to be monomeric.
Together, our SEC-SAXS analysis of the pentad mutant GAPR-1 indicates that at the concentrations tested in this study this mutant exists primarily as a dimer, but equilibrates in a concentration-dependent manner between dimer and monomer oligomeric states that can be detected in SECSAXS.

\section{Discussion}

X-ray crystal structures of GAPR-1 and other CAP-domain homologs have elucidated the protein fold characteristic of 
this family (Fernández et al., 1997; Henriksen et al., 2001; Serrano et al., 2004; Asojo et al., 2005, 2011; Suzuki et al., 2008; Asojo, 2011; Osman et al., 2012; Mason et al., 2014; Darwiche et $a l ., 2016)$. These studies also identified an equatorial surface groove that is present in all homologs of known structure. This groove has been shown to bind metals (Henriksen et al., 2001; Suzuki et al., 2008; Wang et al., 2010; Asojo et al., 2011; Mason et al., 2014; Darwiche et al., 2016), and based on its width, depth and length this groove has also been proposed to be a binding site for peptides (Milne et al., 2003; Osman et al., 2012; Mason et al., 2014). However, to date no direct evidence of peptide binding to this groove has been presented. Our bioinformatics analysis and molecular-docking results suggest that this groove is suitable for binding Beclin 1, and pentad mutation of the conserved GAPR-1 residues H54A/E86A/ G102K/H103A/N138G lining this groove abrogates binding to Beclin 1. These mutations do not impact the GAPR-1 tertiary structure; rather, they alter the size, shape and electrostatic potential of the equatorial groove.

The oligomeric state of WT GAPR-1 has not been unambiguously established because different oligomerization states have been proposed in different studies. A previous study suggested that GAPR-1 exists as a monomer in solution in the absence of lipids (van Galen et al., 2012), while another suggested that it transitions between monomeric and dimeric states in solution (Serrano et al., 2004). Three different crystal structures of GAPR-1, including that reported in this manuscript, indicate that GAPR-1 dimerizes in selective conditions. Further, yeast two-hybrid studies suggest that GAPR-1 may exist as a dimer in cells (Serrano et al., 2004). Interestingly, certain CAP proteins have serine protease activity, yet a canonical active-site triad is only formed upon homodimerization (Milne et al., 2003), suggesting a functional role in the homodimerization of certain CAP domains. The results presented here unambiguously indicate that WT GAPR-1 does not homodimerize in the absence of lipids/other proteins and is a monomer in solution even at SEC injection concentrations of $2 \mathrm{~m} M$. Thus, WT GAPR-1 is likely to form a very weak and transient dimer, which is stabilized at high concentrations such as those used for crystallization and/or in the presence of specific small molecules such as crystallization reagents, selected lipids or $\mathrm{IP}_{6}$. The increased concentration of GAPR-1 at lipid-rich microdomains of the Golgi complex may provide similar conditions in vivo.

Notably, the dimer interface varies in each of the three different GAPR-1 crystal structures determined to date, either owing to mutations, as reported in this study, or to the presence of lipid or $\mathrm{IP}_{6}$ (van Galen et al., 2012). Interestingly, no electron density corresponding to $\mathrm{IP}_{6}$ was seen in the latter structure and thus it is unclear how $\mathrm{IP}_{6}$ mediates changes in dimerization. The crystal structure of the pentad mutant GAPR-1 suggests that the mutations result in subtle changes in structure and residue interactions, which cause an altered mode of dimerization. Together, these studies indicate that GAPR-1 is likely to transition between monomer and different dimer conformations that are dependent on GAPR-1 concentration as well as upon binding of lipids and/or different ligands or protein partners. It is plausible that these different oligomeric states have different mechanisms and impact different biological functions.

Strikingly, the equatorial surface groove is partially occluded by the partner subunit in all three dimeric states crystallized, suggesting that binding partners such as Beclin 1 would not bind to the dimeric state of GAPR-1. Indeed, the interaction between GAPR-1 and Beclin 1 may require colocalization and arrangement of GAPR-1 and Beclin 1 in suitable orientations, which may be facilitated by membrane association in vivo. Further, various cellular factors such as the presence/binding of specific lipids, protein partners and/or post-translational modifications may further regulate this interaction via changes in the conformation and oligomerization of each partner. Binding of GAPR-1 to Beclin 1 BARAD residues 267-284 would sterically obstruct binding of autophagy partners such as UVRAG or ATG14, thereby adversely impacting the assembly of the autophagosome nucleation complex and reducing cellular autophagy levels.

Our model for the binding of GAPR-1 to Beclin 1 BARAD residues 267-284 suggests a mechanism for the disruption of binding caused by the F270S and F274S mutants (ShojiKawata et al., 2013). Our model predicts that Phe270 of Beclin 1 packs against the hydrophobic residue Phe144 in GAPR-1; therefore, the F270S mutation would weaken this hydrophobic packing and the presence of the polar serine hydroxyl group may destabilize the interaction further. The aromatic ring of Phe274 in Beclin 1 packs against the $C^{\beta}$ atom of Ser99 in GAPR-1 with a distance of $3.4 \AA$. An in silico mutation of Phe274 to serine increases the distance between Ser274 in Beclin 1 and Ser99 in GAPR-1 to $5.0 \AA$, abrogating this packing and likely destabilizing the Beclin 1-GAPR-1 interaction.

\section{Conclusion}

The results presented in this study demonstrate that Beclin 1 residues 267-284 are likely to bind to the equatorial groove of GAPR-1, and the conserved residues lining the equatorial binding groove are important for the interaction. Simultaneous mutation of multiple conserved residues such as the H54A/E86A/G102K/H103A/N138G pentad mutation abrogates the GAPR-1-Beclin 1 interaction. We solved the $1.27 \AA$ resolution X-ray crystal structure of this pentad mutant GAPR-1, which shows that the equatorial binding groove of the pentad mutant GAPR-1 is shallower and more positively charged than that of WT GAPR-1. Both WT and pentad mutant GAPR-1 crystallize as dimers, with the equatorial groove of one subunit of the dimer partially occluded by the other, indicating that dimeric GAPR-1 is likely to be unable to bind Beclin 1. In solution, the WT forms a monomer, while the pentad mutant favors the dimeric state. Thus, the reduction in size, the change in shape and the increased positivity of the electrostatic surface of the equatorial binding groove, combined with altered homodimerization resulting in further occlusion of the equatorial groove in the pentad mutant, the increased shape and electrostatic complementarity of the 
homodimer interface and the improved stability of the pentad mutant homodimer relative to that of the WT GAPR-1, are likely to abrogate binding to Beclin 1 .

\section{Acknowledgements}

The authors declare no conflict of interest. The authors are grateful to Dr Lisa Kinch (UT Southwestern Medical Center) for assistance in identifying mutations that may impact GAPR-1 binding to Beclin 1. The authors also thank Dr David Lambright (University of Massachusetts Medical School) for providing access to his program DELA for SVD analysis. Work performed at BioCAT was supported by NIH NIGMS 9P41 GM103622 and use of the PILATUS3 1M detector was funded by NIH NIGMS 1S10OD018090-01. This work incorporates research conducted at the NE-CAT beamlines, which are funded by NIH NIGMS P41 GM103403. This research used resources of the APS, a US DOE Office of Science User Facility operated for the DOE Office of Science by Argonne National Laboratory under Contract No. DE-AC02$06 \mathrm{CH} 11357$.

\section{Funding information}

This work was supported by NIH grants RO3 NS090939 and R15 GM122035 (SCS), U19 AI199725 (BL) and R15 GM113227 (CLC), National Science Foundation grant MCB1413525 (SCS), and NSF and North Dakota EPSCoR grant II1355466 doctoral dissertation awards to MS (PI: SCS).

\section{References}

Aalberts, M., van Dissel-Emiliani, F. M. F., van Adrichem, N. P. H., van Wijnen, M., Wauben, M. H. M., Stout, T. A. E. \& Stoorvogel, W. (2012). Biol. Reprod. 86, 82.

Adams, P. D. et al. (2010). Acta Cryst. D66, 213-221.

Asojo, O. A. (2011). Acta Cryst. D67, 455-462.

Asojo, O. A., Goud, G., Dhar, K., Loukas, A., Zhan, B., Deumic, V., Liu, S., Borgstahl, G. E. O. \& Hotez, P. J. (2005). J. Mol. Biol. 346, 801-814.

Asojo, O. A., Koski, R. A. \& Bonafé, N. (2011). Acta Cryst. D67, 847-855.

Baker, N. A., Sept, D., Joseph, S., Holst, M. J. \& McCammon, J. A. (2001). Proc. Natl Acad. Sci. USA, 98, 10037-10041.

Baxter, R. M., Crowell, T. P., George, J. A., Getman, M. E. \& Gardner, H. (2007). Matrix Biol. 26, 20-29.

Choi, A. M. K., Ryter, S. W. \& Levine, B. (2013). N. Engl. J. Med. 368, 651-662.

Choudhary, V., Darwiche, R., Gfeller, D., Zoete, V., Michielin, O. \& Schneiter, R. (2014). J. Lipid Res. 55, 883-894.

Choudhary, V. \& Schneiter, R. (2012). Proc. Natl Acad. Sci. USA, 109, 16882-16887.

Darwiche, R., Kelleher, A., Hudspeth, E. M., Schneiter, R. \& Asojo, O. A. (2016). Sci. Rep. 6, 28838.

De, S., Krishnadev, O., Srinivasan, N. \& Rekha, N. (2005). BMC Struct. Biol. 5, 15.

Eberle, H. B., Serrano, R. L., Füllekrug, J., Schlosser, A., Lehmann, W. D., Lottspeich, F., Kaloyanova, D., Wieland, F. T. \& Helms, J. B. (2002). J. Cell Sci. 115, 827-838.

Emsley, P., Lohkamp, B., Scott, W. G. \& Cowtan, K. (2010). Acta Cryst. D66, 486-501.

Fernández, C., Szyperski, T., Bruyère, T., Ramage, P., Mösinger, E. \& Wüthrich, K. (1997). J. Mol. Biol. 266, 576-593.
Franke, D., Petoukhov, M. V., Konarev, P. V., Panjkovich, A., Tuukkanen, A., Mertens, H. D. T., Kikhney, A. G., Hajizadeh, N. R., Franklin, J. M., Jeffries, C. M. \& Svergun, D. I. (2017). J. Appl. Cryst. 50, 1212-1225.

Franke, D. \& Svergun, D. I. (2009). J. Appl. Cryst. 42, 342-346.

Galen, J. van, Olrichs, N. K., Schouten, A., Serrano, R. L., Nolte-'t Hoen, E. N. M., Eerland, R., Kaloyanova, D., Gros, P. \& Helms, J. B. (2012). Biochim. Biophys. Acta, 1818, 2175-2183.

Gibbs, G. M., Roelants, K. \& O'Bryan, M. K. (2008). Endocr. Rev. 29, 865-897.

Groves, M. R., Kuhn, A., Hendricks, A., Radke, S., Serrano, R. L., Helms, J. B. \& Sinning, I. (2004). Acta Cryst. D60, 730-732.

Hatok, J. \& Racay, P. (2016). Biomol. Conc. 7, 259-270.

Henriksen, A., King, T. P., Mirza, O., Monsalve, R. I., Meno, K., Ipsen, H., Larsen, J. N., Gajhede, M. \& Spangfort, M. D. (2001). Proteins, 45, 438-448.

Huang, W., Choi, W., Hu, W., Mi, N., Guo, Q., Ma, M., Liu, M., Tian, Y., Lu, P., Wang, F.-L., Deng, H., Liu, L., Gao, N., Yu, L. \& Shi, Y. (2012). Cell Res. 22, 473-489.

Kabsch, W., Kabsch, H. \& Eisenberg, D. (1976). J. Mol. Biol. 100, 283-291.

Kelleher, A., Darwiche, R., Rezende, W. C., Farias, L. P., Leite, L. C. C., Schneiter, R. \& Asojo, O. A. (2014). Acta Cryst. D70, 2186-2196.

Konarev, P. V., Volkov, V. V., Sokolova, A. V., Koch, M. H. J. \& Svergun, D. I. (2003). J. Appl. Cryst. 36, 1277-1282.

Kozin, M. B. \& Svergun, D. I. (2001). J. Appl. Cryst. 34, 33-41.

Krissinel, E. \& Henrick, K. (2007). J. Mol. Biol. 372, 774-797.

Kurcinski, M., Jamroz, M., Blaszczyk, M., Kolinski, A. \& Kmiecik, S. (2015). Nucleic Acids Res. 43, W419-W424.

Lambright, D., Malaby, A. W., Kathuria, S. V., Nobrega, R. P., Bilsel, O., Matthews, C. R., Muthurajan, U., Luger, K., Chopra, R., Irving, T. C. \& Chakravarthy, S. (2014). Trans. Am. Crystallogr. Assoc. 44, 1. http://www.amercrystalassn.org/documents/2013 Transactions/ 1-Chakravarthy.pdf.

Lawrence, M. C. \& Colman, P. M. (1993). J. Mol. Biol. 234, 946950.

Levine, B. \& Klionsky, D. J. (2004). Dev. Cell, 6, 463-477.

Levine, B. \& Kroemer, G. (2008). Cell, 132, 27-42.

Levine, B., Liu, R., Dong, X. \& Zhong, Q. (2015). Trends Cell Biol. 25, 533-544.

Levine, B., Mizushima, N. \& Virgin, H. W. (2011). Nature (London), 469, 323-335.

Maiuri, M., Criollo, A., Tasdemir, E., Vicencio, J., Tajeddine, N., Hickman, J., Geneste, O. \& Kroemer, G. (2007). Autophagy, 3, 374-376.

Malaby, A. W., Chakravarthy, S., Irving, T. C., Kathuria, S. V., Bilsel, O. \& Lambright, D. G. (2015). J. Appl. Cryst. 48, 1102-1113.

Mason, L., Tribolet, L., Simon, A., von Gnielinski, N., Nienaber, L., Taylor, P., Willis, C., Jones, M. K., Sternberg, P. W., Gasser, R. B., Loukas, A. \& Hofmann, A. (2014). Int. J. Biochem. Cell Biol. 50, 146-155.

McCoy, A. J., Grosse-Kunstleve, R. W., Adams, P. D., Winn, M. D., Storoni, L. C. \& Read, R. J. (2007). J. Appl. Cryst. 40, 658-674.

Mei, Y., Glover, K., Su, M. \& Sinha, S. C. (2016). Protein Sci. 25, 17671785.

Milne, T. J., Abbenante, G., Tyndall, J. D. A., Halliday, J. \& Lewis, R. J. (2003). J. Biol. Chem. 278, 31105-31110.

Mizushima, N. (2005). Cell Death Differ. 12, Suppl. 2, 1535-1541.

Mizushima, N. \& Komatsu, M. (2011). Cell, 147, 728-741.

Mizushima, N., Levine, B., Cuervo, A. \& Klionsky, D. (2008). Nature (London), 451, 1069-1075.

Olrichs, N. K. \& Helms, J. B. (2016). AIMS Biophy. 3, 232-246.

Olrichs, N. K., Mahalka, A. K., Kaloyanova, D., Kinnunen, P. K. \& Helms, J. B. (2014). Amyloid, 21, 88-96.

Osman, A., Wang, C. K., Winter, A., Loukas, A., Tribolet, L., Gasser, R. B. \& Hofmann, A. (2012). Biotechnol. Adv. 30, 652-657. 
Pattingre, S., Tassa, A., Qu, X., Garuti, R., Liang, X. H., Mizushima, N., Packer, M., Schneider, M. D. \& Levine, B. (2005). Cell, 122, 927-939.

Rambo, R. \& Tainer, J. (2013). Annu. Rev. Biophys. 42, 415-441.

Richter, S., Wenzel, A., Stein, M., Gabdoulline, R. R. \& Wade, R. C. (2008). Nucleic Acids Res. 36, W276-W280.

Robert, X. \& Gouet, P. (2014). Nucleic Acids Res. 42, W320-W324.

Rubinsztein, D. C., Codogno, P. \& Levine, B. (2012). Nature Rev. Drug Discov. 11, 709-730.

Rubinsztein, D. C., Mariño, G. \& Kroemer, G. (2011). Cell, 146, 682-695.

Serrano, R. L., Kuhn, A., Hendricks, A., Helms, J. B., Sinning, I. \& Groves, M. R. (2004). J. Mol. Biol. 339, 173-183.

Shoji-Kawata, S. et al. (2013). Nature (London), 494, 201-206.

Sinha, S. \& Levine, B. (2009). Oncogene, 27, S137-S148.

Suzuki, N., Yamazaki, Y., Brown, R. L., Fujimoto, Z., Morita, T. \& Mizuno, H. (2008). Acta Cryst. D64, 1034-1042.
Svergun, D. I. (1992). J. Appl. Cryst. 25, 495-503.

Svergun, D., Barberato, C. \& Koch, M. H. J. (1995). J. Appl. Cryst. 28, 768-773.

Theobald, D. L. \& Wuttke, D. S. (2008). PLoS Comput. Biol. 4, e43.

Thompson, J. D., Higgins, D. G. \& Gibson, T. J. (1994). Nucleic Acids Res. 22, 4673-4680.

Van Loon, L. C. \& Van Strien, E. A. (1999). Physiol. Mol. Plant Pathol. 55, 85-97.

Volkov, V. V. \& Svergun, D. I. (2003). J. Appl. Cryst. 36, 860864.

Wang, Y.-L., Kuo, J.-H., Lee, S.-C., Liu, J.-S., Hsieh, Y.-C., Shih, Y.-T., Chen, C.-J., Chiu, J.-J. \& Wu, W.-G. (2010). J. Biol. Chem. 285, 37872-37883.

Winn, M. D. et al. (2011). Acta Cryst. D67, 235-242.

Xu, X., Francischetti, I. M. B., Lai, R., Ribeiro, J. M. C. \& Andersen, J. F. (2012). J. Biol. Chem. 287, 10967-10976. 